\title{
Uniformly Random Attractor for the Three-Dimensional Stochastic Nonautonomous Camassa-Holm Equations
}

\author{
Zhehao Huang and Zhengrong Liu \\ Department of Mathematics, South China University of Technology, Guangzhou 510640, China \\ Correspondence should be addressed to Zhengrong Liu; liuzhr@scut.edu.cn
}

Received 25 November 2012; Revised 3 July 2013; Accepted 17 July 2013

Academic Editor: Ziemowit Popowicz

Copyright (C) 2013 Z. Huang and Z. Liu. This is an open access article distributed under the Creative Commons Attribution License, which permits unrestricted use, distribution, and reproduction in any medium, provided the original work is properly cited.

We consider the uniformly random attractor for the three-dimensional stochastic nonautonomous Camassa-Holm equations in the periodic box $[0, l]^{3}$ in this paper. We associate with the concepts of uniform attractor and random attractor and produce the concept of uniformly random attractor for a process. Then we establish the existence of the uniformly random attractor in $D\left(A^{1 / 2}\right)$ and $D(A)$ for the equations.

\section{Introduction}

The Camassa-Holm equation

$$
u_{t}-u_{x x t}+3 u u_{x}-2 u_{x} u_{x x}-u u_{x x x}=0
$$

models the unidirectional propagation of shallow water waves over a flat bottom [1-4]. It has been paid a large number of attentions due to its rich nonlinear phenomenology. It is completely integrable [1], and it has stable solutions [5]. It possesses the peakons $u=e^{|x-c t|}$ which has been proved stable $[6,7]$. It has been shown that (1) is locally well-posed for initial data $u_{0} \in H^{s}(\mathbb{R})(s>3 / 2)[8,9]$. There are a rich variety of global solutions and blow-up solutions obtained in [8-10]. The global existence of weak solutions, conservative solutions, and disispative solutions was investigated in $[6,11$, 12].

Following the Camassa-Holm (1), some generalized types of the equation have been deeply considered by many authors, for instance [13-18]. The authors in [19] considered the threedimensional Camassa-Holm equations subject to periodic boundary conditions:

$$
\begin{aligned}
& \frac{\partial}{\partial t}\left(\alpha_{0}^{2} u-\alpha_{1}^{2} \Delta u\right)-v \Delta\left(\alpha_{0}^{2} u-\alpha_{1}^{2} \Delta u\right)-u \\
& \quad \times\left(\nabla \times\left(\alpha_{0}^{2} u-\alpha_{1}^{2} \Delta u\right)\right)+\frac{1}{\rho_{0}} \nabla p=f(x),
\end{aligned}
$$

$$
\begin{gathered}
\nabla \cdot u=0, \\
u(x, 0)=u_{0}(x) .
\end{gathered}
$$

They established the global regularity of solutions of the equation and provided the estimates for the Hausdorff and fractal dimensions of the global attractor.

In [20] the authors analyzed the effects produced by stochastic perturbations in the deterministic version of the three-dimensional Lagrangian averaged Navier-Stokes- $\alpha$ model:

$$
\begin{aligned}
& \frac{\partial}{\partial t}(u-\alpha \Delta u)+v(A u-\alpha \Delta(A u)) \\
& +(u \cdot \nabla)(u-\alpha \Delta u)-\alpha \nabla u^{*} \cdot \Delta u+\nabla p \\
& =F(t, u)+G(t, u) \dot{W}(t), \\
& \nabla \cdot u=0, \quad \text { in } D \times(0,+\infty), \\
& u=0, \quad A u=0, \quad \text { on } \partial D \times(0,+\infty), \\
& u(0)=u_{0},
\end{aligned}
$$

that is, the persistence of exponential stability as well as possible stabilization effects produced by the noise. 
In [21] the authors proved the existence of the pullback and forward attractors for three-dimensional Lagrangian averaged Navier-Stokes- $\alpha$ model with delay:

$$
\begin{gathered}
\frac{\partial}{\partial t}(u-\alpha \Delta u)+\nu(A u-\alpha \Delta(A u)) \\
+(u \cdot \nabla)(u-\alpha \Delta u)-\alpha \nabla u^{*} \cdot \Delta u+\nabla p \\
=f(t)+F\left(t, u_{t}\right), \\
\nabla \cdot u=0, \quad \text { in } D \times(\tau,+\infty), \\
u=0, \quad A u=0, \quad \text { on } \partial D \times(\tau,+\infty), \\
u(t)=\phi(t-\tau), \quad \text { in }(\tau-h, \tau), \\
u(\tau)=u_{0} .
\end{gathered}
$$

In [22] the author investigated the existence of finite dimensional uniform attractor for three-dimensional nonautonomous Camassa-Holm equations with periodic boundary conditions:

$$
\begin{gathered}
\frac{\partial}{\partial t}\left(\alpha_{0}^{2} u-\alpha_{1}^{2} \Delta u\right)-v \Delta\left(\alpha_{0}^{2} u-\alpha_{1}^{2} \Delta u\right) \\
-u \times\left(\nabla \times\left(\alpha_{0}^{2} u-\alpha_{1}^{2} \Delta u\right)\right)+\frac{1}{\rho_{0}} \nabla p=f(x, t), \\
\nabla \cdot u=0, \\
u(x, \tau)=u_{\tau}(x) .
\end{gathered}
$$

In $[23,24]$ the author studied the existence of uniform attractor and convergence of the attractor as $\varepsilon \rightarrow 0^{+}$for a nonautonomous three-dimensional Lagrangian averaged Navier-Stokes- $\alpha$ model with singularly oscillating external force:

$$
\begin{gathered}
\frac{\partial}{\partial t}(u-\alpha \Delta u)+v(A u-\alpha \Delta(A u)) \\
+(u \cdot \nabla)(u-\alpha \Delta u)-\alpha \nabla u^{*} \cdot \Delta u+\nabla p=g^{\varepsilon}, \\
\nabla \cdot u=0, \quad \text { in } D \times(\tau,+\infty), \\
u=0, \quad A u=0, \quad \text { on } \partial D \times(\tau,+\infty), \\
u(\tau)=u_{\tau},
\end{gathered}
$$

where

$$
\begin{aligned}
& g^{\varepsilon}(x, y, z, t) \\
& = \begin{cases}g_{0}(x, y, z, t)+\frac{1}{\varepsilon^{\rho}} g_{1}(x, y, z, t), & \varepsilon \in(0,1], \rho \in[0,1), \\
g_{0}(x, y, z, t), & \varepsilon=0 .\end{cases}
\end{aligned}
$$

Motivated by all their works, we initial our work to investigate the equations perturbed by an additive noise. We consider the following viscous version of three-dimensional stochastic nonautonomous Camassa-Holm equation in the periodic box $[0, l]^{3}$ :

$$
\begin{gathered}
\frac{\partial}{\partial t}\left(\alpha_{0}^{2} u-\alpha_{1}^{2} \Delta u\right)-v \Delta\left(\alpha_{0}^{2} u-\alpha_{1}^{2} \Delta u\right) \\
-u \times\left(\nabla \times\left(\alpha_{0}^{2} u-\alpha_{1}^{2} \Delta u\right)\right)+\frac{1}{\rho_{0}} \nabla p \\
=f(x, t)+Q W_{t}, \\
\nabla \cdot u=0, \\
u(x, \tau)=u_{\tau}(x),
\end{gathered}
$$

where $p / \rho_{0}=\pi / \rho_{0}+\alpha_{0}^{2}|u|^{2}-\alpha_{1}^{2}(u \cdot \Delta u)$ is the modified pressure, while $\pi$ is the pressure, $v>0$ is the constant viscosity, and $\rho_{0}>0$ is a constant density. The function $f$ is a given body forcing, and $\alpha_{0}>0, \alpha_{1} \geq 0$ are scale parameters. $W(t)$ are two-sided real-valued Wiener processes on a probability space which will be specified later. $Q: \mathbb{R}^{n} \rightarrow L^{2}\left([0, l]^{3}\right)^{3}$ is a bounded linear operator. Also observing that at the limiting case $\alpha_{0}=1, \alpha_{1}=0$, we obtain the three-dimensional stochastic Navier-Stokes with periodic boundary conditions.

Attractor is an important concept to describe the longtime behavior of solutions for a system in mathematical physics [25-27]. The notion of uniform attractor parallelling to that of the global autonomous systems has been systematically considered in [26]. In the approach presented in [27], to construct the uniform attractor, instead of the associated process $\left\{\mathscr{U}_{\sigma}(t, \tau), t \geq \tau, \tau \in \mathbb{R}\right\}$, one should consider a family of processes $\left\{\mathscr{U}_{\sigma}(t, s)\right\}, \sigma \in \Sigma$, in some Banach space $E$ where the functional parameter $\sigma_{0}(s), s \in \mathbb{R}$, is called the symbol and $\Sigma$ is the symbol space including $\sigma_{0}(s)$. The approach implies that the structure of uniform attractor is described by the representation as a union of sections of all kernels of the family of processes. The kernel is the set of all complete trajectories of a process.

While in the real world, a system is usually uncertain due to some external noise, which is random. The random effects are considered not only as compensations for the defects in some deterministic models but rather essential phenomena [28-32]. In order to capture the essential dynamics of random dynamical systems with wide fluctuations, the concept of pullback random attractor was introduced in $[29,33,34]$, as an extension to stochastic systems of the theory of attractors for deterministic systems in $[25,27,35-43]$. A pullback random attractor $\mathscr{A}(\omega)$ which can be constructed by a closed random absorbing set $\mathscr{K}(\omega)$ for an asymptotically compact stochastic mapping $\mathcal{S}(t, s, \omega)$ is given by

$$
\mathscr{A}(\omega)=\bigcap_{s \geq \tau} \overline{\bigcup_{t \geq s}} \mathcal{S}\left(t, \tau, \theta_{\tau-t} \omega, \mathscr{K}\left(\theta_{\tau-t} \omega\right)\right),
$$

where $\theta_{t}$ is the metric process on probability space. The existence of random attractors for stochastic dynamical systems has been investigated extensively by many authors $[29,33,34,44-49]$. In our paper, we associate with the concepts of uniform attractor and random attractor together 
and give the concept of uniformly random attractor. Then we consider (8) in an appropriate space and show that there is a uniformly (with respect to $f$ ) random attractor $\mathscr{A}(\omega)$ which all solutions approach as $t \rightarrow \infty$. To our best knowledge, the long-time dynamical behavior of the three-dimensional stochastic nonautonomous Camassa-Holm equations has not been discussed, and we believe that it is a significant work to obtain a uniformly (with respect to $f$ ) random attractor for the system.

The paper is organized as follows. In Section 2, we present the abstract results describing the uniformly random attractor and some relevant definitions. In Section 3, we give some functional settings which are foundations for us to obtain the existence of uniformly (with respect to $f$ ) random attractor of (8). In Section 4, we convert (8) with an additive noise to deterministic equations with random parameters and define a process corresponding to the equations. In Section 5, we obtain the existence of uniformly (with respect to $f$ ) random attractors for (8) on the basis of the above preparations.

\section{Abstract Results}

In this section, we associate with the concepts of uniform attractor and random attractor and obtain the notion of uniformly random attractor. Let $\left(E,\|\cdot\|_{E}\right)$ be a separable Hilbert space with the Borel $\sigma$-algebra $\mathscr{B}(E)$, and let $(\Omega, \mathscr{F}, \mathbb{P})$ be a probability space.

Definition 1. $\left(\theta_{t}\right)_{t \in \mathbb{R}}$ is called a measurable flow on probability space $(\Omega, \mathscr{F})$ if $\theta: \mathbb{R} \times \Omega \rightarrow \Omega$ is $(\mathbb{R} \times \mathscr{F}, \mathscr{F})$-measurable, $\theta_{0}$ is the identity on $\Omega, \theta_{s+t}=\theta_{t} \circ \theta_{s}$ for all $s, t \in \mathbb{R}$, and $\theta_{t} \mathbb{P}=\mathbb{P}$ for all $t \in \mathbb{R}$.

Definition 2. A random bounded set $\{B(\omega)\}_{\omega \in \Omega}$ of $E$, is called tempered with respect to $\left(\theta_{t}\right)_{t \in \mathbb{R}}$ if for $\mathbb{P}$ a.e. $\omega \in \Omega$,

$$
\lim _{t \rightarrow \infty} e^{-\beta t} d\left(B\left(\theta_{-t} \omega\right)\right)=0 \quad \forall \beta>0,
$$

where $d(B)=\sup _{x \in E}\|x\|_{E}$.

Definition 3. A random set $\{\mathscr{K}(\omega)\}_{\omega \in \Omega}$ is called an absorbing set of a stochastic mapping $\mathcal{S}(t, s, \omega)$ in $E$ if for every random bounded set $B$ and $\mathbb{P}$ a.e. $\omega \in \Omega$, there exists $t_{B}(\omega)>0$ such that

$$
\mathcal{S}\left(t, s, \theta_{s-t} \omega\right) B\left(\theta_{s-t}(\omega)\right) \subseteq \mathscr{K}(\omega) \quad \forall t \geq t_{B}(\omega) .
$$

Let $\{\mathcal{U}(t, \tau, \omega)\}=\{\mathcal{U}(t, \tau, \omega), t \geq \tau, t, \tau \in \mathbb{R}, \omega \in \Omega\}$ be a three-parameter family of mappings acting on $E$ :

$$
\mathcal{U}(t, \tau, \omega): E \longrightarrow E, \quad t \geq \tau, t, \tau \in \mathbb{R}, \omega \in \Omega .
$$

Definition 4. A three-parameter family of random mappings $\{\mathscr{U}(t, \tau, \omega)\}$ is said to be a process in $E$ if it is $\left(\mathscr{B}\left(\mathbb{R}^{+}\right) \times\right.$ $\left.\mathscr{B}\left(\mathbb{R}^{+}\right) \times \mathscr{F} \times \mathscr{B}(E), \mathscr{B}(E)\right)$-measurable and for $\mathbb{P}$ a.e. $\omega \in \Omega$ it satisfies

$$
\begin{gathered}
\mathcal{U}\left(t, s, \theta_{s-\tau} \omega\right) \circ \mathcal{U}(s, \tau, \omega)=\mathcal{U}(t, \tau, \omega), \\
\forall t \geq s \geq \tau, \tau \in \mathbb{R}, \\
\mathcal{U}(\tau, \tau, \omega)=I d, \quad \tau \in \mathbb{R} .
\end{gathered}
$$

Definition 5. A family of processes $\mathscr{U}_{\sigma}(t, \tau, \omega), \sigma \in \Sigma$, acting in $E$ is said to be $(E \times \Sigma, E)$-continuous, if, for $\mathbb{P}$ a.e. $\omega \in \Omega$ and fixed $t, \tau, t \geq \tau$, the random mapping $(u, \sigma) \mapsto \mathcal{U}_{\sigma}(t, \tau, \omega) u$ is continuous from $E \times \sum$ into $E$.

Definition 6. A random curve $u(s, \omega), s \in \mathbb{R}$, is said to be a complete trajectory of the process $\{\mathcal{U}(t, \tau, \omega)\}$ if for $\mathbb{P}$ a.e. $\omega \in \Omega$

$$
\{\mathcal{U}(t, \tau, \omega)\} u(\tau, \omega)=u(t, \omega), \quad \forall t \geq \tau, t, \tau \in \mathbb{R} .
$$

Definition 7. The random kernel $\mathcal{N}(\omega)$ of the process $\{\mathcal{U}(t, \tau, \omega)\}$ consists of all bounded complete trajectories of the process $\{\mathscr{U}(t, \tau, \omega)\}$ :

$$
\begin{array}{r}
\mathcal{N}(\omega)=\{u(\cdot, \omega) \mid u(\cdot, \omega) \text { satisfies Definition } 6 \\
\text { and } \left.\|u(s, \omega)\|_{E} \leq M_{u}(\omega) \text { for } s \in \mathbb{R}\right\} .
\end{array}
$$

Definition 8 . The random set

$$
\mathcal{N}(s, \omega)=\{u(s, \omega) \mid u(\cdot, \omega) \in \mathcal{N}(\omega)\} \subseteq E
$$

is said to be the random kernel section at time $t=s, s \in \mathbb{R}$.

Let $B_{t}(\omega)=\bigcup_{\sigma \in \Sigma} \bigcup_{s \geq t} \mathscr{U}_{\sigma}\left(s, t, \theta_{t-s} \omega\right) B\left(\theta_{t-s} \omega\right)$, where $B(\omega)$ is a random set. Denote that $\overline{B(\omega)}$ is closure of the set $B(\omega)$ and $\mathbb{R}_{\tau}=\{t \in \mathbb{R} \mid t \geq \tau\}$.

Definition 9. A random set $\mathscr{W}_{\tau, \Sigma}(B)(\omega)=\bigcap_{t \geq \tau} \overline{B_{t}(\omega)}$ is called the uniformly (with respect to $\sigma \in \Sigma$ ) random (pullback) omega-limit set of $B(\omega)$ which can be characterized as follows, analogously to that for semigroups,

$$
\begin{gathered}
y \in \mathscr{W}_{\tau, \Sigma}(B)(\omega) \Longleftrightarrow \text { there are sequences }\left\{x_{n}\right\} \subset B\left(\theta_{\tau-t_{n}} \omega\right), \\
\left\{\sigma_{n}\right\} \subset \Sigma,\left\{t_{n}\right\} \subset \mathbb{R}_{\tau},
\end{gathered}
$$$$
\text { such that } t_{n} \longrightarrow+\infty \text { and }
$$$$
\mathscr{U}_{\sigma_{n}}\left(t_{n}, \tau, \theta_{\tau-t_{n}} \omega\right) x_{n} \longrightarrow y \quad(n \longrightarrow \infty)
$$

Let $B(\omega) \in \mathscr{B}(E)$, and its Kuratowski measure of noncompactness $\kappa(B)$ is defined by

$$
\begin{aligned}
\kappa(B)=\inf \{\delta>0 \mid B \text { admits a finit } \\
\text { covering by sets of diameter } \leq \delta\} .
\end{aligned}
$$

Definition 10. A family of processes $\left\{\mathcal{U}_{\sigma}(t, \tau, \omega)\right\}, \sigma \in \Sigma$, is said to be uniformly (with respect to $\sigma \in \Sigma$ ) random (pullback) omega-limit compact if, for $\mathbb{P}$ a.e. $\omega \in \Omega$ and any $\tau \in \mathbb{R}$, the set $B_{t}(\omega)$ is bounded for every $t$ and $\lim _{t \rightarrow \infty} \kappa\left(B_{t}(\omega)\right)=0$.

We now present a method to verify the uniformly (with respect to $\sigma \in \Sigma$ ) random (pullback) omega-limit compactness.

Definition 11. A family of processes $\left\{\mathcal{U}_{\sigma}(t, \tau, \omega)\right\}, \sigma \in \Sigma$, is said to satisfy uniformly (with respect to $\sigma \in \Sigma$ ) condition 
(C) if, for $\mathbb{P}$ a.e. $\omega \in \Omega$ and any fixed $\tau \in \mathbb{R}$, tempered set $B(\omega) \in \mathscr{B}(E), \varepsilon>0$, there exist $t_{0}=t(\tau, B, \varepsilon, \omega) \geq \tau$ and $\mathrm{a}$ finite dimensional subspace $E_{1}$ of $E$ such that

(i) $\Pi\left(\bigcup_{\sigma \in \Sigma} \bigcup_{t \geq t_{0}} \mathcal{U}_{\sigma}\left(t, \tau, \theta_{\tau-t} \omega\right) B\left(\theta_{\tau-t} \omega\right)\right)$ is bounded,
and

(ii) $\left\|(I-\Pi)\left(\bigcup_{\sigma \in \Sigma} \bigcup_{t \geq t_{0}} \mathscr{U}_{\sigma}\left(t, \tau, \theta_{\tau-t} \omega\right) x\right)\right\| \leq \varepsilon, \forall x \in$ $B\left(\theta_{\tau-t} \omega\right)$,

where $\Pi: E \rightarrow E_{1}$ is a bounded projector.

Therefore, we have the following results.

Theorem 12. Let $\Sigma$ be a metric space, and let $T(t)$ be a continuous invariant semigroup $T(t) \Sigma=\Sigma$ on $\Sigma$. A family of processes $\{\mathcal{U}(t, \tau, \omega)\}, \sigma \in \Sigma$, acting in $E$ is $(E \times \Sigma, E)$ continuous (weakly) and possesses the compact uniformly (with respect to $\sigma \in \Sigma$ ) random attractor $\mathscr{A}_{\Sigma}(\omega)$ satisfying

$$
\begin{aligned}
\mathscr{A}_{\Sigma}(\omega) & =\mathscr{W}_{0, \Sigma}\left(B_{0}(\omega)\right)=\mathscr{W}_{\tau, \Sigma}\left(B_{0}(\omega)\right) \\
& =\bigcup_{\sigma \in \Sigma} \mathcal{N}_{\sigma}(0, \omega), \quad \forall \tau \in \mathbb{R},
\end{aligned}
$$

if it

(i) has a bounded uniformly (with respect to $\sigma \in \Sigma$ ) random absorbing set $B_{0}(\omega)$, and

(ii) satisfies uniformly (with respect to $\sigma \in \Sigma$ ) condition (C).

Moreover, if $E$ is a uniformly convex Banach space, then the converse is true.

\section{Functional Setting}

We consider the probability space $(\Omega, \mathscr{F}, \mathbb{P})$ where

$$
\Omega=\left\{\omega=\left(\omega_{1}, \omega_{2}, \ldots, \omega_{n}\right)^{T} \in C\left(\mathbb{R}, \mathbb{R}^{n}\right): \omega(0)=0\right\} .
$$

$\mathscr{F}$ is the Borel $\sigma$-algebra induced by the compact-open topology of $\Omega$ and $\mathbb{P}$ the corresponding Wiener measure on $(\Omega, \mathscr{F})$. Then we will identify $W(t)$ with

$$
W(t)=\left(w_{1}(t), w_{2}(t), \ldots, w_{n}(t)\right)^{T}=\omega(t) \quad \text { for } t \in \mathbb{R} .
$$

Define the time shift by

$$
\theta_{t} \omega(\cdot)=\omega(\cdot+t)-\omega(t), \quad \omega \in \Omega, t \in \mathbb{R} .
$$

Then $\left\{\theta_{t}\right\}_{t \in \mathbb{R}}$ is a family of measure preserving transformations on probability space $(\Omega, \mathscr{F}, \mathbb{P})$ in Definition 1 .

Next we define a symbol space $\Sigma\left(\sigma_{0}\right)$ for $(8)$. We assume that the function $f(\cdot, t)=: f(t) \in L_{\text {loc }}^{2}(\mathbb{R} ; E)$ is translation bounded. That is, for $f(s) \in L_{b}^{2}(\mathbb{R} ; E)$, we have

$$
\|f\|_{L_{b}^{2}}^{2}=\|f\|_{L_{b}^{2}(\mathbb{R} ; E)}^{2}=\sup _{t \in \mathbb{R}} \int_{t}^{t+1}\|f(s)\|_{E}^{2} d s<\infty .
$$

Definition 13 (cf. [41]). A function $\varphi \in L_{\text {loc }}^{2}(\mathbb{R} ; E)$ is said to be normal if, for any $\varepsilon>0$, there exists $\delta>0$ such that

$$
\sup _{t \in \mathbb{R}} \int_{t}^{t+\delta}\|\varphi(s)\|_{E}^{2} d s \leq \varepsilon .
$$

We denote by $L_{n}^{2}(\mathbb{R} ; E)$ the set of all normal functions in $L_{\text {loc }}^{2}(\mathbb{R} ; E)$. Obviously, $L_{n}^{2}(\mathbb{R} ; E) \subset L_{b}^{2}(\mathbb{R} ; E)$, and it is proved in [41] that $L_{n}^{2}(\mathbb{R} ; E)$ is a closed subset of $L_{b}^{2}(\mathbb{R} ; E)$. Let a fixed symbol $\sigma_{0}(s)=f_{0}(s)=f_{0}(\cdot, s)$ be normal functions in $L_{\text {loc }}^{2}(\mathbb{R} ; E)$. That is, the family of translation $\left\{f_{0}(s+\eta), \eta \in\right.$ $\mathbb{R}\}$ forms a normal function set in $L_{\text {loc }}^{2}\left(\left[T_{1}, T_{2}\right] ; E\right)$, where $\left[T_{1}, T_{2}\right]$ is an arbitrary interval of the time axis $\mathbb{R}$. Therefore,

$$
\Sigma\left(\sigma_{0}\right)=\Sigma\left(f_{0}\right)=\left\{f_{0}(x, s+\eta) \mid \eta \in \mathbb{R}\right\}_{L_{\mathrm{loc}}^{2}(\mathbb{R} ; E)} .
$$

After integrating (8), one can easily see that

$$
\frac{d}{d t} \int_{[0, l]^{3}}\left(\alpha_{0}^{2} u-\alpha_{1}^{2} \Delta u\right) d x=\int_{[0, l]^{3}} f d x+\int_{[0, l]^{3}} Q W_{t} d x .
$$

On the other hand, because of the spatial periodicity of solution, we have $\int_{[0, l]^{3}} \Delta u d x=0$. Then we have

$$
\frac{d}{d t} \int_{[0, l]^{3}} \alpha_{0}^{2} u d x=\int_{[0, l]^{3}} f d x+\int_{[0, l]^{3}} Q W_{t} d x .
$$

That is, the mean of solution is invariant provided that the means of the forcing term and the perturbing term are zero. In this paper, we will consider the forcing term, perturbing term and initial values with spatial means that are zero. That is, we assume $\int_{[0, l]^{3}} f d x=0, \int_{[0, l]^{3}} Q W_{t} d x=0$, and $\int_{[0, l]^{3}} u_{\tau} d x=0$.

Next we introduce some essential functional spaces.

(i) We denote by $\mathscr{V}=\{\varphi: \varphi$ a vector-valued trigonometric polynomial defined on $[0, l]^{3}$, such that $\nabla \cdot \varphi=0$ and $\left.\int_{[0, l]^{3}} \varphi(x) d x=0\right\}$, and we let $H$ and $V$ be the closure of $\mathscr{V}$ in $L^{2}\left([0, l]^{3}\right)^{3}$ and in $H^{1}\left([0, l]^{3}\right)^{3}$, respectively. We can observe that $H^{\perp}$, the orthogonal complement of $H$ in $L^{2}\left([0, l]^{3}\right)^{3}$, is $\{\nabla p$ : $\left.p \in H^{1}\left([0, l]^{3}\right)\right\}$ (cf. $\left.[38,41]\right)$.

(ii) We denote by $P: L^{2}\left([0, l]^{3}\right)^{3} \rightarrow H$ the $L^{2}$ orthogonal projection, usually referred to as Helmholtz-Leray projector, and by $A=-P \Delta$ the Stokes operator with domain $D(A)=H^{2}\left([0, l]^{3}\right)^{3} \cap V$. Note that, in the case of periodic boundary condition, $A=-\left.\Delta\right|_{D(A)}$ is a self-adjoint positive operator with compact inverse. Hence, the space $H$ has an orthogonal basis $\left\{e_{j}\right\}_{j}^{\infty}$ of eigenfunctions of $A$, that is, $A e_{j}=\lambda_{j} e_{j}$, with

$$
\begin{array}{r}
0<\lambda_{1} \leq \lambda_{2} \leq \cdots \leq \lambda_{j} \leq \cdots, \\
\lambda_{j} \rightarrow+\infty, \text { as } j \rightarrow \infty
\end{array}
$$

In fact, these eigenvalues have the form $|k|^{2} 4 \pi / L^{2}$ with $k \in \mathbb{Z}^{3} /\{0\}$. 
(iii) We denote by $(\cdot, \cdot)$ the $L^{2}\left([0, l]^{3}\right)^{3}$ inner product and by $\|\cdot\|_{2}$ the corresponding $L^{2}\left([0, l]^{3}\right)^{3}$ norm. By virtue of Poincaré inequality one can show that there is a constant $c>0$, such that

$$
\begin{gathered}
c\|A u\|_{2} \leq\|u\|_{H^{2}} \leq c^{-1}\|A u\|_{2} \\
\text { for every } u \in D(A), \\
c\left\|A^{1 / 2} u\right\|_{2} \leq\|u\|_{H^{1}} \leq c^{-1}\left\|A^{1 / 2} u\right\|_{2}
\end{gathered}
$$

for every $u \in V$.

Moreover, one can show that $V=D\left(A^{1 / 2}\right)$ (cf. [38, 42]). We denote by $V^{\prime}$ the dual of $V$. Hereafter $c$ will denote a generic scale invariant positive constant which is independent of the physical parameters in the equation.

(iv) Following the notation for the Navier-Stokes equations we denote yhat $B(u, v)=P[(u \cdot \nabla) v]$, and we set $B(v) u=B(u, v)$ for every $u, v \in V$. That is, for every fixed $v \in V, B(v)$ is a linear operator acting on $u$. Note that

$$
\begin{gathered}
(B(u, v), w)=-(B(u, w), v) \\
\text { for every } u, v, w \in V .
\end{gathered}
$$

We also denote that $\widetilde{B}(u, v)=-P(u \times(\nabla \times v))$ for every $u, v \in$ $V$. Using the identity

$$
(b \cdot \nabla) a+\sum_{j=1}^{3} a_{j} \nabla b_{j}=-b \times(\nabla \times a)+\nabla(a \cdot b),
$$

one can easily show that

$$
\begin{gathered}
(\widetilde{B}(u, v), w)=(B(u, v), w)-(B(w, v), u) \\
=\left(B(v) u-B^{*}(v) u, w\right)
\end{gathered}
$$

for every $u, v, w \in V$, where $B^{*}(v)$ denotes the adjoint operator of the linear operator $B(v)$ defined above. As a result we have

$$
\widetilde{B}(u, v)=\left(B(v)-B^{*}(v)\right) u \quad \text { for every } u, v \in V .
$$

The next lemma will present some properties of the bilinear operator $\widetilde{B}$.

Lemma 14 (cf. [19]). The operator $\widetilde{B}$ can be extended continuously from $V \times V$ with values in $V^{\prime}$, and it satisfies

$$
\begin{aligned}
& \mid\langle\widetilde{B}(u, v), w\rangle_{V^{\prime}} \leq c\|u\|_{2}^{1 / 2}\left\|A^{1 / 2} u\right\|_{2}^{1 / 2}\left\|A^{1 / 2} v\right\|_{2}\left\|A^{1 / 2} w\right\|_{2}, \\
& \mid\langle\widetilde{B}(u, v), w\rangle_{V^{\prime}} \leq c\left\|A^{1 / 2} u\right\|_{2}\left\|A^{1 / 2} v\right\|_{2}\|w\|_{2}^{1 / 2}\left\|A^{1 / 2} w\right\|_{2}^{1 / 2}
\end{aligned}
$$

for every $u, v, w \in V$. Moreover,

$$
\langle\widetilde{B}(u, v), w\rangle_{V^{\prime}}=-\langle\widetilde{B}(w, v), u\rangle_{V^{\prime}}, \quad \text { for every } u, v, w \in V
$$

and in particular

$$
\langle\widetilde{B}(u, v), u\rangle_{V^{\prime}} \equiv 0 \quad \text { for every } u, v \in V .
$$

Furthermore, one has

$$
\left|\langle\widetilde{B}(u, v), w\rangle_{D(A)^{\prime}}\right| \leq c\|u\|_{2}\left\|A^{1 / 2} v\right\|_{2}\left\|A^{1 / 2} w\right\|_{2}^{1 / 2}\|A w\|_{2}^{1 / 2}
$$

for every $u \in H, v \in V$, and $w \in D(A)$ and by symmetry one has

$$
\left|\langle\widetilde{B}(u, v), w\rangle_{D(A)^{\prime}}\right| \leq c\left\|A^{1 / 2} u\right\|_{2}^{1 / 2}\|A u\|_{2}^{1 / 2}\left\|A^{1 / 2} v\right\|_{2}\|w\|_{2}
$$

for every $u \in D(A), v \in V$, and $w \in H$. Also

$$
\begin{aligned}
& \left|\langle\widetilde{B}(u, v), w\rangle_{D(A)^{\prime}}\right| \\
& \leq c\left(\|u\|_{2}^{1 / 2}\left\|A^{1 / 2} u\right\|_{2}^{1 / 2}\|v\|_{2}\|A w\|_{2}\right. \\
& \left.\quad+\|v\|_{2}\left\|A^{1 / 2} u\right\|_{2}\left\|A^{1 / 2} w\right\|_{2}^{1 / 2}\|A w\|_{2}^{1 / 2}\right)
\end{aligned}
$$

for every $u \in V, v \in H$, and $w \in D(A)$. In addition,

$$
\begin{gathered}
\left|\langle\widetilde{B}(u, v), w\rangle_{D(A)^{\prime}}\right| \\
\leq c\left(\left\|A^{1 / 2} u\right\|_{2}^{1 / 2}\|A u\|_{2}^{1 / 2}\|v\|_{2}\left\|A^{1 / 2} w\right\|_{2}\right. \\
\left.+\|v\|_{2}\|A u\|_{2}\|w\|_{2}^{1 / 2}\left\|A^{1 / 2} w\right\|_{2}^{1 / 2}\right)
\end{gathered}
$$

for every $u \in D(A), v \in H$, and $w \in V$.

\section{Stochastic Nonautonomous Camassa-Holm Equations}

We now apply the result in Section 2 to the stochastic nonautonomous Camassa-Holm equations. To associate a family of processes $\mathscr{U}_{\sigma}(t, s, \omega)$ with the stochastic equations over $(\Sigma, T(t))$ and $\left(\Omega, \mathscr{F}, \mathbb{P},\left(\theta_{t}\right)_{t \in \mathbb{R}}\right)$, we need to convert the stochastic equations with a random additive term into deterministic equations with a random parameter.

First we define the bounded linear operator $Q$ in (8) as follows:

$$
\mathrm{Q} x=\sum_{j=1}^{n} x_{j} h_{j}, \quad x=\left(x_{1}, x_{2}, \ldots, x_{n}\right)^{T} \in \mathbb{R}^{n},
$$

where $h_{j} \in D(A), j=1,2, \ldots, n$.

Given $j=1,2, \ldots, n$, consider the Ornstein-Uhlenbeck equation:

$$
d y_{j}+\mu y_{j} d t=d w_{j}(t) .
$$

One can easily check that a solution to (42) is given by

$$
y_{j}(t)=y_{j}\left(\theta_{t} \omega_{j}\right)=-\mu \int_{-\infty}^{0} e^{\mu \tau}\left(\theta_{t} \omega_{j}\right)(\tau) d \tau, \quad t \in \mathbb{R} .
$$


It is known that the random variable $\left|y_{j}\left(\omega_{j}\right)\right|$ is tempered and that $y_{j}\left(\theta_{t} \omega_{j}\right)$ is $\mathbb{P}$ a.e is continuous. Now we put $z\left(\theta_{t} \omega\right)=\left(\alpha_{0}^{2}+\right.$ $\left.\alpha_{1}^{2} A\right)^{-1} \sum_{j=1}^{m} h_{j} y_{j}\left(\theta_{t} \omega_{j}\right)$. By (42) we have

$$
\begin{aligned}
& d\left(\alpha_{0}^{2} z\left(\theta_{t} \omega\right)+\alpha_{1}^{2} A z\left(\theta_{t} \omega\right)\right) \\
& \quad+\mu\left(\alpha_{0}^{2} z\left(\theta_{t} \omega\right) d t+\alpha_{1}^{2} A z\left(\theta_{t} \omega\right)\right) d t \\
& =\sum_{j=1}^{n} h_{j} d w_{j}(t) .
\end{aligned}
$$

Employing Cauchy-Schwarz's inequality, we get

$$
\begin{gathered}
\left\|z\left(\theta_{t} \omega\right)\right\|_{2}^{2}+\left\|A^{1 / 2} z\left(\theta_{t} \omega\right)\right\|_{2}^{2}+\left\|A z\left(\theta_{t} \omega\right)\right\|_{2}^{2} \\
\leq c \sum_{j=1}^{n}\left|y_{j}\left(\theta_{t} \omega_{j}\right)\right|^{2}
\end{gathered}
$$

To show that $(8)$ corresponds to a process $\left\{\mathscr{U}_{\sigma}(t, \tau, \omega)\right\}$, we let $v=u-z\left(\theta_{t} \omega\right)$, where $u$ is a solution of (8). Then for $v(t, \omega)$, we have

$$
\begin{aligned}
\frac{\partial}{\partial t}\left(\alpha_{0}^{2} v+\alpha_{1}^{2} A v\right) \\
\quad+v A\left(\alpha_{0}^{2} v+\alpha_{1}^{2} A v+\alpha_{0}^{2} z\left(\theta_{t} \omega\right)+\alpha_{1}^{2} A z\left(\theta_{t} \omega\right)\right) \\
\quad-\left(v+z\left(\theta_{t} \omega\right)\right) \\
\quad \times\left(\nabla \times\left(\alpha_{0}^{2} v+\alpha_{1}^{2} A v+\alpha_{0}^{2} z\left(\theta_{t} \omega\right)+\alpha_{1}^{2} A z\left(\theta_{t} \omega\right)\right)\right) \\
\quad+\frac{1}{\rho_{0}} \nabla p \\
=f(x, t)+\mu \alpha_{0}^{2} z\left(\theta_{t} \omega\right)+\mu \alpha_{1}^{2} A z\left(\theta_{t} \omega\right)
\end{aligned}
$$

defined in the periodic box $[0, l]^{3}$, satisfying

$$
\begin{gathered}
\nabla \cdot v=0, \\
v(x, \tau, \omega)=v_{\tau}(x, \omega)=u_{\tau}(x)-z\left(\theta_{\tau} \omega\right) \in H .
\end{gathered}
$$

We apply $P$ to (46) and use the notation in Section 3 to obtain the equivalent system of equations

$$
\begin{gathered}
\frac{\partial}{\partial t}\left(\alpha_{0}^{2} v+\alpha_{1}^{2} A v\right) \\
+\nu A\left(\alpha_{0}^{2} v+\alpha_{1}^{2} A v+\alpha_{0}^{2} z\left(\theta_{t} \omega\right)+\alpha_{1}^{2} A z\left(\theta_{t} \omega\right)\right) \\
+\widetilde{B}\left(v+z\left(\theta_{t} \omega\right), \alpha_{0}^{2} v+\alpha_{1}^{2} A v\right. \\
\left.+\alpha_{0}^{2} z\left(\theta_{t} \omega\right)+\alpha_{1}^{2} A z\left(\theta_{t} \omega\right)\right) \\
=\operatorname{Pf}(x, t)+\mu \alpha_{0}^{2} z\left(\theta_{t} \omega\right)+\mu \alpha_{1}^{2} A z\left(\theta_{t} \omega\right)
\end{gathered}
$$

satisfying the initial condition

$$
v(x, \tau, \omega)=v_{\tau}(x, \omega) .
$$

By a Galerkin method as in [19], it can be proved that if $f(x, t) \in L_{\text {loc }}^{2}((0, T) ; H)$ and $v_{\tau} \in V$, for $\mathbb{P}$ a.e. $\omega \in \Omega$, (48) has a unique solution satisfying for any $T>\tau$

$$
\begin{gathered}
v\left(t, \omega, v_{\tau}\right) \in C([\tau, T) ; V) \bigcap L^{2}([\tau, T) ; D(A)), \\
\frac{d v}{d t} \in L^{2}([\tau, T) ; H)
\end{gathered}
$$

and such that, for almost all $t \in[\tau, T)$ and for any $w \in D(A)$,

$$
\begin{aligned}
& \left\langle\frac{d}{d t}\left(\alpha_{0}^{2} v+\alpha_{1}^{2} A v\right), w\right\rangle_{D(A)^{\prime}} \\
& +v\left\langleA \left(\alpha_{0}^{2} v+\alpha_{1}^{2} A v\right.\right. \\
& \left.\left.\quad+\alpha_{0}^{2} z\left(\theta_{t} \omega\right)+\alpha_{1}^{2} A z\left(\theta_{t} \omega\right)\right), w\right\rangle_{D(A)^{\prime}} \\
& +\left\langle\widetilde { B } \left( v+z\left(\theta_{t} \omega\right), \alpha_{0}^{2} v+\alpha_{1}^{2} A v\right.\right. \\
& \left.\left.\quad+\alpha_{0}^{2} z\left(\theta_{t} \omega\right)+\alpha_{1}^{2} A z\left(\theta_{t} \omega\right)\right), w\right\rangle_{D(A)^{\prime}} \\
& =\left(P f(x, t)+\mu \alpha_{0}^{2} z\left(\theta_{t} \omega\right)+\mu \alpha_{1}^{2} A z\left(\theta_{t} \omega\right), w\right) .
\end{aligned}
$$

Now for any $f(x, t) \in \Sigma\left(f_{0}\right),(48)$ with $f$ instead of $f_{0}$ possesses a corresponding process $\left\{\mathcal{U}_{f}(t, \tau, \omega)\right\}$ acting on $V$. It is analogous to the proof in [27] to prove that, for $\mathbb{P}$ a.e $\omega \in \Omega$, the family $\left\{\mathcal{U}_{f}(t, \tau, \omega) \mid f \in \Sigma\left(f_{0}\right)\right\}$ of processes is $\left(V \times \Sigma\left(f_{0}\right) ; V\right)$-continuous. Let

$$
\mathscr{N}_{f}(\omega)=\left\{v_{f}(x, t, \omega) \text { for } t \in \mathbb{R} \mid v_{f}(x, t, \omega)\right.
$$$$
\text { is solution of (48) satisfying }
$$

$$
\left.\left\|v_{f}(x, t, \omega)\right\|_{H} \leq M_{f}(\omega) \forall t \in \mathbb{R}\right\}
$$

be the so-called kernel of the process $\left\{\mathscr{U}_{f}(t, \tau, \omega)\right\}$.

\section{Uniformly Random Attractor for Stochastic Nonautonomous Camassa-Holm Equation}

In [19], the authors have shown that the semigroup corresponding to the autonomous system possesses a global attractor. In [21-24], the authors have proved that the deterministic version of nonautonomous system has a uniform attractor. The main objective of this section is to obtain the existence of uniformly (with respect to $f \in \Sigma\left(f_{0}\right)$ ) random attractor for the stochastic nonautonomous Camassa-Holm equations in $V$ and $D(A)$.

Lemma 15. Let $\{B(\omega)\}_{\omega \in \Omega}$ be tempered and $v_{\tau}(\omega) \in B(\omega)$. Then the process $\left\{\mathcal{U}_{f}(t, \tau, \omega)\right\}$ corresponding to (48) possesses a uniformly (with respect to $f \in \Sigma\left(f_{0}\right)$ ) random absorbing set $\mathscr{K}_{0}(\omega)$ in $V$. 
Proof. Letting $w=v$ in (51), we have

$$
\begin{aligned}
\frac{1}{2} \frac{d}{d t}( & \left.\alpha_{0}^{2}\|v\|_{2}^{2}+\alpha_{1}^{2}\left\|A^{1 / 2} v\right\|_{2}^{2}\right)+v\left(\alpha_{0}^{2}\left\|A^{1 / 2} v\right\|_{2}^{2}+\alpha_{1}^{2}\|A v\|_{2}^{2}\right) \\
= & -v\left(\sum_{j=1}^{n} A h_{j} y_{j}\left(\theta_{t} \omega_{j}\right), v\right) \\
& -\left(\widetilde{B}\left(v+z\left(\theta_{t} \omega\right), \alpha_{0}^{2} v+\alpha_{1}^{2} A v+\sum_{j=1}^{n} h_{j} y_{j}\left(\theta_{t} \omega_{j}\right)\right), v\right) \\
& +(P f(x, t), v)+\left(\sum_{j=1}^{n} \mu h_{j} y_{j}\left(\theta_{t} \omega_{j}\right), v\right) .
\end{aligned}
$$

Now we estimate the second term of (53) on the right-hand side. Applying Lemma 14 we have

$$
\begin{aligned}
& \left|\left(\widetilde{B}\left(v+z\left(\theta_{t} \omega\right), \alpha_{0}^{2} v+\alpha_{1}^{2} A v+\sum_{j=1}^{n} h_{j} y_{j}\left(\theta_{t} \omega_{j}\right)\right), v\right)\right| \\
& =\left|\left(\widetilde{B}\left(z\left(\theta_{t} \omega\right), \alpha_{0}^{2} v+\alpha_{1}^{2} A v+\sum_{j=1}^{n} h_{j} y_{j}\left(\theta_{t} \omega_{j}\right)\right), v\right)\right| \\
& \leq c\left\|A^{1 / 2} z\left(\theta_{t} \omega\right)\right\|_{2}^{1 / 2}\left\|A z\left(\theta_{t} \omega\right)\right\|_{2}^{1 / 2} \\
& \times\left\|\alpha_{0}^{2} v+\alpha_{1}^{2} A v+\sum_{j=1}^{n} h_{j} y_{j}\left(\theta_{t} \omega_{j}\right)\right\|_{2}\left\|A^{1 / 2} v\right\|_{2} \\
& +c\left\|A z\left(\theta_{t} \omega\right)\right\|_{2}\left\|\alpha_{0}^{2} v+\alpha_{1}^{2} A v+\sum_{j=1}^{n} h_{j} y_{j}\left(\theta_{t} \omega_{j}\right)\right\|_{2} \\
& \times\|v\|_{2}^{1 / 2}\left\|A^{1 / 2} v\right\|_{2}^{1 / 2} \\
& \leq c\left\|A^{1 / 2} z\left(\theta_{t} \omega\right)\right\|_{2}\|v\|_{2}^{2}+c\left\|A z\left(\theta_{t} \omega\right)\right\|_{2}\left\|A^{1 / 2} v\right\|_{2}^{2} \\
& +\frac{v \alpha_{1}^{2}}{4}\|A v\|_{2}^{2} \\
& +c\left\|A^{1 / 2} z\left(\theta_{t} \omega\right)\right\|_{2}\left\|A z\left(\theta_{t} \omega\right)\right\|_{2}\left\|A^{1 / 2} v\right\|_{2}^{2} \\
& +c\left\|A^{1 / 2} z\left(\theta_{t} \omega\right)\right\|_{2}\left\|A z\left(\theta_{t} \omega\right)\right\|_{2} \\
& +c \sum_{j=1}^{n}\left|y_{j}\left(\theta_{t} \omega_{j}\right)\right|^{2}\left\|A^{1 / 2} v\right\|_{2}^{2} \\
& +c\left\|A z\left(\theta_{t} \omega\right)\right\|_{2}\|v\|_{2}^{2}+\frac{v \alpha_{1}^{2}}{4}\|A v\|_{2}^{2} \\
& +c\left\|A z\left(\theta_{t} \omega\right)\right\|_{2}^{2}\|v\|_{2}^{2}+c\left\|A z\left(\theta_{t} \omega\right)\right\|_{2}^{2}\left\|A^{1 / 2} v\right\|_{2}^{2} \\
& +c\left\|A z\left(\theta_{t} \omega\right)\right\|_{2}^{2}+c \sum_{j=1}^{n}\left|y_{j}\left(\theta_{t} \omega_{j}\right)\right|^{2}\left\|A^{1 / 2} v\right\|_{2}^{2}
\end{aligned}
$$

$$
\begin{aligned}
& +c \sum_{j=1}^{n}\left|y_{j}\left(\theta_{t} \omega_{j}\right)\right|^{2} \\
\leq & \frac{\nu \alpha_{1}^{2}}{2}\|A v\|_{2}^{2}+c \sum_{j=1}^{n}\left|y_{j}\left(\theta_{t} \omega_{j}\right)\right|\left(\alpha_{0}^{2}\|v\|_{2}^{2}+\alpha_{1}^{2}\left\|A^{1 / 2} v\right\|_{2}^{2}\right) \\
& +c \sum_{j=1}^{n}\left|y_{j}\left(\theta_{t} \omega_{j}\right)\right|^{2}\left(\alpha_{0}^{2}\|v\|_{2}^{2}+\alpha_{1}^{2}\left\|A^{1 / 2} v\right\|_{2}^{2}\right) \\
& +c \sum_{j=1}^{n}\left|y_{j}\left(\theta_{t} \omega_{j}\right)\right|^{2} .
\end{aligned}
$$

By Poincarés inequality, we have

$$
\begin{gathered}
v\left|\left(\sum_{j=1}^{n} A h_{j} y_{j}\left(\theta_{t} \omega_{j}\right), v\right)\right| \leq \frac{v \alpha_{0}^{2}}{6}\left\|A^{1 / 2} v\right\|_{2}^{2}+c \sum_{j=1}^{n}\left|y_{j}\left(\theta_{t} \omega_{j}\right)\right|^{2}, \\
|(P f(x, t), v)| \leq\|f\|_{V^{\prime}}\|v\|_{2} \leq \frac{v \alpha_{0}^{2}}{6}\left\|A^{1 / 2} v\right\|_{2}^{2}+c\|f\|_{V^{\prime}}^{2}, \\
\left|\left(\sum_{j=1}^{n} \mu h_{j} y_{j}\left(\theta_{t} \omega_{j}\right), v\right)\right| \leq \frac{v \alpha_{0}^{2}}{6}\left\|A^{1 / 2} v\right\|_{2}^{2}+c \sum_{j=1}^{n}\left|y_{j}\left(\theta_{t} \omega_{j}\right)\right|^{2} .
\end{gathered}
$$

Associating with the above inequalities and employing Poincaré's inequality, we have

$$
\begin{aligned}
& \frac{d}{d t}\left(\alpha_{0}^{2}\|v\|_{2}^{2}+\alpha_{1}^{2}\left\|A^{1 / 2} v\right\|_{2}^{2}\right) \\
& \quad+v \lambda_{1}\left(\alpha_{0}^{2}\|v\|_{2}^{2}+\alpha_{1}^{2}\left\|A^{1 / 2} v\right\|_{2}^{2}\right) \\
& \leq c \sum_{j=1}^{n}\left|y_{j}\left(\theta_{t} \omega_{j}\right)\right|\left(\alpha_{0}^{2}\|v\|_{2}^{2}+\alpha_{1}^{2}\left\|A^{1 / 2} v\right\|_{2}^{2}\right) \\
& +c \sum_{j=1}^{n}\left|y_{j}\left(\theta_{t} \omega_{j}\right)\right|^{2}\left(\alpha_{0}^{2}\|v\|_{2}^{2}+\alpha_{1}^{2}\left\|A^{1 / 2} v\right\|_{2}^{2}\right) \\
& \quad+c \sum_{j=1}^{n}\left|y_{j}\left(\theta_{t} \omega_{j}\right)\right|^{2}+c\|f\|_{V^{\prime}}^{2} .
\end{aligned}
$$

Applying Gronwall's lemma, we have

$$
\begin{aligned}
& \alpha_{0}^{2}\|v(t, \omega)\|_{2}^{2}+\alpha_{1}^{2}\left\|A^{1 / 2} v(t, \omega)\right\|_{2}^{2} \\
& \leq e^{J_{1}(\tau, t, \omega)}\left(\alpha_{0}^{2}\left\|v_{\tau}(\omega)\right\|_{2}^{2}+\alpha_{1}^{2}\left\|A^{1 / 2} v_{\tau}(\omega)\right\|_{2}^{2}\right) \\
& \quad+\int_{\tau}^{t}\left(c \sum_{j=1}^{n}\left|y_{j}\left(\theta_{s} \omega_{j}\right)\right|^{2}+c\|f(s)\|_{V^{\prime}}^{2}\right) e^{J_{1}(s, t, \omega)} d s,
\end{aligned}
$$


where

$$
\begin{aligned}
J_{1}(\tau, t, \omega)= & -\nu \lambda_{1} t+\nu \lambda_{1} \tau+c \sum_{j=1}^{n} \int_{\tau}^{t}\left|y_{j}\left(\theta_{s} \omega_{j}\right)\right| d s \\
& +c \sum_{j=1}^{n} \int_{\tau}^{t}\left|y_{j}\left(\theta_{s} \omega_{j}\right)\right|^{2} d s .
\end{aligned}
$$

Replacing $\omega$ by $\theta_{-t} \omega$ in (57) and (58), we have

$$
\begin{aligned}
& \alpha_{0}^{2}\left\|v\left(t, \theta_{-t} \omega\right)\right\|_{2}^{2}+\alpha_{1}^{2}\left\|A^{1 / 2} v\left(t, \theta_{-t} \omega\right)\right\|_{2}^{2} \\
& \leq e^{J_{1}(\tau-t, 0, \omega)}\left(\alpha_{0}^{2}\left\|v_{\tau}\left(\theta_{-t} \omega\right)\right\|_{2}^{2}+\alpha_{1}^{2}\left\|A^{1 / 2} v_{\tau}\left(\theta_{-t} \omega\right)\right\|_{2}^{2}\right) \\
& \quad+\int_{\tau-t}^{0}\left(c \sum_{j=1}^{n}\left|y_{j}\left(\theta_{s} \omega_{j}\right)\right|^{2}+c\|f(s+t)\|_{V^{\prime}}^{2}\right) e^{J_{1}(s, 0, \omega)} d s .
\end{aligned}
$$

Note that $\left\{\left|y_{j}\left(\theta_{t} \omega_{j}\right)\right|\right\}_{j=1}^{n}$ are stationary and ergodic (cf. [24]), then it follows from ergodic theorem that

$$
\lim _{t \rightarrow \infty} \frac{1}{t} \int_{-t}^{0}\left|y_{j}\left(\theta_{\tau} \omega_{j}\right)\right| d \tau=E\left(\left|y_{j}\left(\omega_{j}\right)\right|\right)
$$

On the other hand, we have

$$
\begin{aligned}
& \lim _{t \rightarrow \infty} \frac{1}{t} \int_{-t}^{0}\left|y_{j}\left(\theta_{\tau} \omega_{j}\right)\right| d \tau=E\left(\left|y_{j}\left(\omega_{j}\right)\right|\right)=\frac{c}{\sqrt{\mu}}, \\
& \lim _{t \rightarrow \infty} \frac{1}{t} \int_{-t}^{0}\left|y_{j}\left(\theta_{\tau} \omega_{j}\right)\right|^{2} d \tau=E\left(\left|y_{j}\left(\omega_{j}\right)\right|^{2}\right)=\frac{c}{\mu} .
\end{aligned}
$$

Employing (61), we have

$$
\begin{aligned}
\lim _{s \rightarrow-\infty} \frac{J_{1}(s, 0, \omega)}{s}= & \lim _{s \rightarrow-\infty}\left(\nu \lambda_{1}+c \sum_{j=1}^{m} \frac{1}{s} \int_{s}^{0}\left|y_{j}\left(\theta_{\tau} \omega_{j}\right)\right| d \tau\right. \\
& \left.+c \sum_{j=1}^{m} \frac{1}{s} \int_{s}^{0}\left|y_{j}\left(\theta_{\tau} \omega_{j}\right)\right|^{2} d \tau\right) \\
& =\left(\nu \lambda_{1}-\frac{c}{\sqrt{\mu}}-\frac{c}{\mu}\right) .
\end{aligned}
$$

So for $\mathbb{P}$ a.e. $\omega \in \Omega$, there are $T_{0}(\omega)>0$ and $\mu_{0}>0$ such that, for $s \geq T_{1}(\omega)$ and $\mu \geq \mu_{0}$,

$$
\begin{gathered}
\frac{J_{1}(s, 0, \omega)}{s}=\nu \lambda_{1}+c \sum_{j=1}^{m} \frac{1}{s} \int_{s}^{0}\left|y_{j}\left(\theta_{\tau} \omega_{j}\right)\right| d \tau \\
+c \sum_{j=1}^{m} \frac{1}{s} \int_{s}^{0}\left|y_{j}\left(\theta_{\tau} \omega_{j}\right)\right|^{2} d \tau<0, \\
J_{1}(s, 0, \omega) \longrightarrow-\infty, \quad \text { as } s \longrightarrow-\infty .
\end{gathered}
$$

Since $\left\{\left|y_{j}\left(\omega_{j}\right)\right|\right\}_{j=1}^{n}$ are tempered, the integral

$$
r_{1}(\omega)=\int_{-\infty}^{0} \sum_{j=1}^{n}\left|y_{j}\left(\theta_{s} \omega_{j}\right)\right|^{2} e^{J_{1}(s, 0, \omega)} d s
$$

is convergent. For any $t>\tau$, there exists $K \in \mathbb{N}$ such that $-K<\tau-t$, and we have

$$
\begin{aligned}
\int_{\tau-t}^{0}\|f(s+t)\|_{V^{\prime}}^{2} e^{J_{1}(s, 0, \omega)} d s \\
\quad=\int_{\tau-t}^{0}\left\|f_{0}(s+t+\eta)\right\|_{V^{\prime}}^{2} e^{J_{1}(s, 0, \omega)} d s \\
\quad \leq \int_{-K}^{0}\left\|f_{0}(s+t+\eta)\right\|_{V^{\prime}}^{2} e^{J_{1}(s, 0, \omega)} d s .
\end{aligned}
$$

Then by piecewise integration, we have

$$
\begin{aligned}
& \int_{-K}^{0}\left\|f_{0}(s+t+\eta)\right\|_{V^{\prime}}^{2} e^{J_{1}(s, 0, \omega)} d s \\
& \quad=\sum_{j=1}^{K} \int_{-n+j-1}^{-n+j}\left\|f_{0}(s+t+\eta)\right\|_{V^{\prime}}^{2} e^{J_{1}(s, 0, \omega)} d s .
\end{aligned}
$$

By differential mean value theorem, for each $1 \leq j \leq K$, there exists $s_{j} \in[-n+j-1,-n+j]$, such that

$$
\begin{aligned}
& \sum_{j=1}^{K} \int_{-n+j-1}^{-n+j}\left\|f_{0}(s+t+\eta)\right\|_{V^{\prime}}^{2} e^{J_{1}(s, 0, \omega)} d s \\
& =\sum_{j=1}^{K} e^{J_{1}\left(s_{j}, 0, \omega\right)} \int_{-n+j-1}^{-n+j}\left\|f_{0}(s+t+\eta)\right\|_{V^{\prime}}^{2} d s .
\end{aligned}
$$

By the property of normal function, there exists $M_{f_{0}}$, which just depends on $f_{0}$, such that

$$
\sup _{t \in \mathbb{R}} \int_{t}^{t+1}\left\|f_{0}(s)\right\|_{V^{\prime}}^{2} d s<M_{f_{0}}
$$

It is easy to check that the series $\sum_{j=1}^{\infty} e^{J_{1}\left(s_{j}, 0, \omega\right)}$ is convergent. Associating with (65)-(68), for any $t>\tau$, we have

$$
\int_{\tau-t}^{0}\|f(s+t)\|_{V^{\prime}}^{2} e^{J_{1}(s, 0, \omega)} d s \leq r_{2}(\omega),
$$

where

$$
r_{2}(\omega)=M_{f_{0}} \sum_{j=1}^{\infty} e^{J_{1}\left(s_{j}, 0, \omega\right)} .
$$

Therefore (64) and (69) imply that, for $t>\tau$, the second term on the right-hand side of (59) can be bounded by

$$
\int_{\tau-t}^{0}\left(c \sum_{j=1}^{n}\left|y_{j}\left(\theta_{s} \omega_{j}\right)\right|^{2}+c\|f(s+t)\|_{V^{\prime}}^{2}\right) e^{J_{1}(s, 0, \omega)} d s \leq r_{3}(\omega),
$$

where $r_{3}(\omega)=c r_{1}(\omega)+c r_{2}(\omega)$. For $v_{\tau}(\omega) \in B(\omega)$ being tempered, there exists $T_{1}(\omega)>0$ such that, when $t \geq T_{2}(\omega)$,

$$
e^{J_{1}(\tau-t, 0, \omega)}\left(\alpha_{0}^{2}\left\|v_{\tau}\left(\theta_{-t} \omega\right)\right\|_{2}^{2}+\alpha_{1}^{2}\left\|A^{1 / 2} v_{\tau}\left(\theta_{-t} \omega\right)\right\|_{2}^{2}\right)<r_{3}(\omega) .
$$


Letting $T_{3}(\omega)=\max \left\{T_{1}(\omega), T_{2}(\omega)\right\}$ and $r_{4}(\omega)=2 r_{3}(\omega)$, when $t \geq T_{3}(\omega)$, we have

$$
\begin{array}{r}
\alpha_{0}^{2}\left\|v\left(t, \theta_{-t} \omega\right)\right\|_{2}^{2}+\alpha_{1}^{2}\left\|A^{1 / 2} v\left(t, \theta_{-t} \omega\right)\right\|_{2}^{2} \leq r_{4}(\omega), \\
\forall t \geq T_{3}(\omega), f \in \Sigma\left(f_{0}\right) .
\end{array}
$$

We define

$$
\begin{aligned}
\mathscr{K}_{1}(\omega)= & \left\{v(\omega) \in V \mid \alpha_{0}^{2}\|v(\omega)\|_{2}^{2}\right. \\
& \left.+\alpha_{1}^{2}\left\|A^{1 / 2} v(\omega)\right\|_{2}^{2} \leq r_{4}(\omega)\right\} .
\end{aligned}
$$

In conclusion, $\mathscr{K}_{1}(\omega)$ is a uniformly random absorbing set for $\{\mathscr{U}(t, \tau, \omega)\}$ in $V$, which complete the proof.

Lemma 16. Let $\{B(\omega)\}_{\omega \in \Omega}$ be tempered and $v_{\tau}(\omega) \in B(\omega)$. Then the process $\left\{\mathscr{U}_{f}(t, \tau, \omega)\right\}$ corresponding to (48) possesses a uniformly (with respect to $f \in \Sigma\left(f_{0}\right)$ ) random absorbing set $\mathscr{K}_{2}(\omega)$ in $D(A)$.

Proof. Integrating (56) into $[t, t+1]$ where $t \geq T_{3}(\omega)$, we have

$$
\begin{aligned}
& \alpha_{0}^{2}\|v(t+1, \omega)\|_{2}^{2}+\alpha_{1}^{2}\left\|A^{1 / 2} v(t+1, \omega)\right\|_{2}^{2} \\
& +v \int_{t}^{t+1}\left(\alpha_{0}^{2}\left\|A^{1 / 2} v(s, \omega)\right\|_{2}^{2}+\alpha_{1}^{2}\|A v(s, \omega)\|_{2}^{2}\right) d s \\
& \leq\left(\alpha_{0}^{2}\|v(t, \omega)\|_{2}^{2}+\alpha_{1}^{2}\left\|A^{1 / 2} v(t, \omega)\right\|_{2}^{2}\right) \\
& +c \sum_{j=1}^{n} \int_{t}^{t+1}\left|y_{j}\left(\theta_{s} \omega_{j}\right)\right| \\
& \quad \times\left(\alpha_{0}^{2}\|v(s, \omega)\|_{2}^{2}+\alpha_{1}^{2}\left\|A^{1 / 2} v(s, \omega)\right\|_{2}^{2}\right) d s \\
& +c \sum_{j=1}^{n} \int_{t}^{t+1}\left|y_{j}\left(\theta_{s} \omega_{j}\right)\right|^{2} \\
& \quad \times\left(\alpha_{0}^{2}\|v(s, \omega)\|_{2}^{2}+\alpha_{1}^{2}\left\|A^{1 / 2} v(s, \omega)\right\|_{2}^{2}\right) d s \\
& +c \sum_{j=1}^{n} \int_{t}^{t+1}\left|y_{j}\left(\theta_{s} \omega_{j}\right)\right|^{2} d s .
\end{aligned}
$$

Replacing $\omega$ by $\theta_{-t-1} \omega$ in (75), we have

$$
\begin{array}{r}
v \int_{t}^{t+1}\left(\alpha_{0}^{2}\left\|A^{1 / 2} v\left(s, \theta_{-t-1} \omega\right)\right\|_{2}^{2}+\alpha_{1}^{2}\left\|A v\left(s, \theta_{-t-1} \omega\right)\right\|_{2}^{2}\right) d s \\
\leq c \sum_{j=1}^{n} \int_{t}^{t+1}\left|y_{j}\left(\theta_{s-t-1} \omega_{j}\right)\right|\left(\alpha_{0}^{2}\left\|v\left(s, \theta_{-t-1} \omega\right)\right\|_{2}^{2}\right. \\
\left.+\alpha_{1}^{2}\left\|A^{1 / 2} v\left(s, \theta_{-t-1} \omega\right)\right\|_{2}^{2}\right) d s
\end{array}
$$

$$
\begin{aligned}
& +c \sum_{j=1}^{n} \int_{t}^{t+1}\left|y_{j}\left(\theta_{s-t-1} \omega_{j}\right)\right|^{2}\left(\alpha_{0}^{2}\left\|v\left(s, \theta_{-t-1} \omega\right)\right\|_{2}^{2}\right. \\
& \left.\quad+\alpha_{1}^{2}\left\|A^{1 / 2} v\left(s, \theta_{-t-1} \omega\right)\right\|_{2}^{2}\right) d s \\
& +c \sum_{j=1}^{n} \int_{t}^{t+1}\left|y_{j}\left(\theta_{s-t-1} \omega_{j}\right)\right|^{2} d s \\
& +\left(\alpha_{0}^{2}\left\|v\left(t, \theta_{-t-1} \omega\right)\right\|_{2}^{2}+\alpha_{1}^{2}\left\|A^{1 / 2} v\left(t, \theta_{-t-1} \omega\right)\right\|_{2}^{2}\right) .
\end{aligned}
$$

As the previous consideration in Lemma 15 , for $s \in[t, t+1)$ where $t \geq T_{2}(\omega)$, we have

$$
\alpha_{0}^{2}\left\|v\left(s, \theta_{-t-1} \omega\right)\right\|_{2}^{2}+\alpha_{1}^{2}\left\|A^{1 / 2} v\left(s, \theta_{-t-1} \omega\right)\right\|_{2}^{2} \leq r_{5}(\omega),
$$

where $r_{5}(\omega)=e^{\nu \lambda_{1}} r_{4}(\omega)$.

Associating (76) with (77), we have

$$
\begin{aligned}
\int_{t}^{t+1} & \left(\alpha_{0}^{2}\left\|A^{1 / 2} v\left(s, \theta_{-t-1} \omega\right)\right\|_{2}^{2}+\alpha_{1}^{2}\left\|A v\left(s, \theta_{-t-1} \omega\right)\right\|_{2}^{2}\right) d s \\
& \leq r_{6}(\omega),
\end{aligned}
$$

where

$$
\begin{aligned}
r_{6}(\omega)= & c r_{5}(\omega) \sum_{j=1}^{n} \int_{-1}^{0}\left|y_{j}\left(\theta_{s} \omega_{j}\right)\right| d s \\
& +c r_{5}(\omega) \sum_{j=1}^{n} \int_{-1}^{0}\left|y_{j}\left(\theta_{s} \omega_{j}\right)\right|^{2} d s \\
& +c \sum_{j=1}^{n} \int_{-1}^{0}\left|y_{j}\left(\theta_{s} \omega_{j}\right)\right|^{2} d s+r_{5}(\omega) .
\end{aligned}
$$

Now we let $w=A v$ in (51), and we have

$$
\begin{aligned}
& \frac{1}{2} \frac{d}{d t}\left(\alpha_{0}^{2}\left\|A^{1 / 2} v\right\|_{2}^{2}+\alpha_{1}^{2}\|A v\|_{2}^{2}\right) \\
& +v\left(\alpha_{0}^{2}\|A v\|_{2}^{2}+\alpha_{1}^{2}\left\|A^{3 / 2} v\right\|_{2}^{2}\right) \\
& =-v\left(\sum_{j=1}^{n} A h_{j} y_{j}\left(\theta_{t} \omega_{j}\right), A v\right) \\
& -\left(\widetilde { B } \left(v+z\left(\theta_{t} \omega\right), \alpha_{0}^{2} v+\alpha_{1}^{2} A v\right.\right. \\
& \left.\left.+\sum_{j=1}^{n} h_{j} y_{j}\left(\theta_{t} \omega_{j}\right)\right), A v\right) \\
& +(P f(x, t), A v)+\left(\sum_{j=1}^{n} \mu h_{j} y_{j}\left(\theta_{t} \omega_{j}\right), A v\right) .
\end{aligned}
$$


Employing Lemma 14, the bilinear term in (80) can be bounded by

$$
\begin{aligned}
& \left(\widetilde{B}\left(v+z\left(\theta_{t} \omega\right), \alpha_{0}^{2} v+\alpha_{1}^{2} A v+\sum_{j=1}^{n} h_{j} y_{j}\left(\theta_{t} \omega_{j}\right)\right), A v\right) \\
& \leq c\left(\left\|A^{1 / 2} v+A^{1 / 2} z\left(\theta_{t} \omega\right)\right\|_{2}^{1 / 2}\left\|A v+A z\left(\theta_{t} \omega\right)\right\|_{2}^{1 / 2}\right. \\
& \left.\quad \times\left\|\alpha_{0}^{2} v+\alpha_{1}^{2} A v+\sum_{j=1}^{n} h_{j} y_{j}\left(\theta_{t} \omega_{j}\right)\right\|_{2}\left\|A^{3 / 2} v\right\|_{2}\right) \\
& +c\left(\left\|A v+A z\left(\theta_{t} \omega\right)\right\|_{2}\left\|_{0}^{2} v+\alpha_{1}^{2} A v+\sum_{j=1}^{n} h_{j} y_{j}\left(\theta_{t} \omega_{j}\right)\right\|_{2}\right. \\
& +c \sum_{j=1}^{n}\left|y_{j}\left(\theta_{t} \omega_{j}\right)\right|^{4} \cdot \\
& +c \sum_{j=1}^{n}\left|y_{j}\left(\theta_{t} \omega_{j}\right)\right|^{2}\left(\alpha_{0}^{2}\left\|A^{1 / 2} v\right\|_{2}^{2}+\alpha_{0}^{2}\|A v\|_{2}^{2}\right) \\
& +c\left(\alpha_{0}^{2}\|v\|_{2}^{2}+\alpha_{0}^{2}\left\|A^{1 / 2} v\right\|_{2}^{2}\right)\left(y_{j}^{2}\left\|A^{1 / 2} v\right\|_{2}^{2}+\alpha_{0}^{2}\|A v\|_{2}^{2}\right) \\
& \left.+c\|A v\|_{2}^{1 / 2}\left\|A^{3 / 2} v\right\|_{2}^{1 / 2}\right) \\
& +c\left(\alpha_{0}^{2}\|v\|_{2}^{2}+\alpha_{0}^{2}\left\|A^{3 / 2} v\right\|_{2}^{2}+\frac{v \alpha_{0}^{2}}{4}\|A v\|_{2}^{2}\right. \\
& +c\left(\alpha_{0}^{2}\left\|A^{1 / 2} v\right\|_{2}^{2}\|v\|_{2}^{2}+\alpha_{0}^{2}\left\|A^{1 / 2} v\right\|_{2}^{2}\right)
\end{aligned}
$$

The other terms are bounded by

$$
\begin{gathered}
\nu\left|\left(\sum_{j=1}^{n} A h_{j} y_{j}\left(\theta_{t} \omega_{j}\right), A v\right)\right| \leq \frac{v \alpha_{0}^{2}}{4}\|A v\|_{2}^{2}+c \sum_{j=1}^{n}\left|y_{j}\left(\theta_{t} \omega_{j}\right)\right|^{2} . \\
|(P f(x, t), A v)| \leq\|f\|_{V^{\prime}}\|A v\|_{2} \leq \frac{v \alpha_{0}^{2}}{4}\|A v\|_{2}^{2}+c\|f\|_{V^{\prime}}^{2} . \\
\mu\left|\left(\sum_{j=1}^{n} h_{j} y_{j}\left(\theta_{t} \omega_{j}\right), A v\right)\right| \leq \frac{v \alpha_{0}^{2}}{4}\|A v\|_{2}^{2}+c \sum_{j=1}^{n}\left|y_{j}\left(\theta_{t} \omega_{j}\right)\right|^{2} .
\end{gathered}
$$

Associating with all the above inequalities, we have

$$
\begin{aligned}
& \frac{d}{d t}\left(\alpha_{0}^{2}\left\|A^{1 / 2} v\right\|_{2}^{2}+\alpha_{1}^{2}\|A v\|_{2}^{2}\right) \\
& \leq c\left(\alpha_{0}^{2}\|v\|_{2}^{2}+\alpha_{1}^{2}\left\|A^{1 / 2} v\right\|_{2}^{2}\right)^{2} \\
& +c\left(\alpha_{0}^{2}\left\|A^{1 / 2} v\right\|_{2}^{2}+\alpha_{1}^{2}\|A v\|_{2}^{2}\right)^{2} \\
& +c \sum_{j=1}^{n}\left|y_{j}\left(\theta_{t} \omega_{j}\right)\right|^{2} \\
& +c \sum_{j=1}^{n}\left|y_{j}\left(\theta_{t} \omega_{j}\right)\right|^{4} \\
& +c\left(\alpha_{0}^{2}\|v\|_{2}^{2}+\alpha_{1}^{2}\left\|A^{1 / 2} v\right\|_{2}^{2}\right) \\
& \quad \times\left(\alpha_{0}^{2}\left\|A^{1 / 2} v\right\|_{2}^{2}+\alpha_{1}^{2}\|A v\|_{2}^{2}\right) \\
& +c \sum_{j=1}^{n}\left|y_{j}\left(\theta_{t} \omega_{j}\right)\right|^{2}\left(\alpha_{0}^{2}\|v\|_{2}^{2}+\alpha_{1}^{2}\left\|A^{1 / 2} v\right\|_{2}^{2}\right) \\
& +c\|f\|_{V^{\prime}}^{2} \\
& +c \sum_{j=1}^{n}\left|y_{j}\left(\theta_{t} \omega_{j}\right)\right|^{2}\left(\alpha_{0}^{2}\left\|A^{1 / 2} v\right\|_{2}^{2}+\alpha_{1}^{2}\|A v\|_{2}^{2}\right)
\end{aligned}
$$

Applying Gronwall's lemma on $[s, t+1]$ where $s \in(t, t+1)$ and $t \geq T_{3}(\omega)$, we have

$$
\begin{aligned}
& \alpha_{0}^{2}\left\|A^{1 / 2} v(t+1, \omega)\right\|_{2}^{2}+\alpha_{1}^{2}\|A v(t+1, \omega)\|_{2}^{2} \\
& \leq\left(\alpha_{0}^{2}\left\|A^{1 / 2} v(s, \omega)\right\|_{2}^{2}+\alpha_{1}^{2}\|A v(s, \omega)\|_{2}^{2}\right) e^{J_{2}(s, t+1, \omega)} \\
& \quad+c \int_{s}^{t+1} \sum_{j=1}^{n}\left|y_{j}\left(\theta_{\tau} \omega_{j}\right)\right|^{2} e^{J_{2}(\tau, t+1, \omega)} d \tau \\
& +c \int_{s}^{t+1} \sum_{j=1}^{n}\left|y_{j}\left(\theta_{\tau} \omega_{j}\right)\right|^{4} e^{J_{2}(\tau, t+1, \omega)} d \tau \\
& +c \int_{s}^{t+1}\|f(\tau)\|_{V^{\prime}}^{2} e^{J_{2}(\tau, t+1, \omega)} d \tau \\
& +c \int_{s}^{t+1} \sum_{j=1}^{n}\left|y_{j}\left(\theta_{\tau} \omega_{j}\right)\right|^{2} \\
& \quad \times\left(\alpha_{0}^{2}\|v(\tau, \omega)\|_{2}^{2}+\alpha_{1}^{2}\left\|A^{1 / 2} v(\tau, \omega)\right\|_{2}^{2}\right) e^{J_{2}(\tau, t+1, \omega)} d \tau \\
& +c \int_{s}^{t+1}\left(\alpha_{0}^{2}\|v(\tau, \omega)\|_{2}^{2}+\alpha_{1}^{2}\left\|A^{1 / 2} v(\tau, \omega)\right\|_{2}^{2}\right)^{2} e^{J_{2}(\tau, t+1, \omega)} d \tau
\end{aligned}
$$


where

$$
\begin{aligned}
J_{2}(s, t, \omega) & \\
= & c \int_{s}^{t}\left(\alpha_{0}^{2}\|v(\tau, \omega)\|_{2}^{2}+\alpha_{1}^{2}\left\|A^{1 / 2} v(\tau, \omega)\right\|_{2}^{2}\right) d \tau \\
& +c \int_{s}^{t}\left(\alpha_{0}^{2}\left\|A^{1 / 2} v(\tau, \omega)\right\|_{2}^{2}+\alpha_{1}^{2}\|A v(\tau, \omega)\|_{2}^{2}\right) d \tau \\
& +c \int_{s}^{t} \sum_{j=1}^{n}\left|y_{j}\left(\theta_{\tau} \omega_{j}\right)\right|^{2} d \tau .
\end{aligned}
$$

Replacing $\omega$ by $\theta_{-t-1} \omega$ in (84) and (85), we have

$$
\begin{aligned}
& J_{2}\left(\tau, t+1, \theta_{-t-1} \omega\right) \\
& \leq c \int_{t}^{t+1}\left(\alpha_{0}^{2}\left\|v\left(\tau, \theta_{-t-1} \omega\right)\right\|_{2}^{2}\right. \\
& \left.\quad+\alpha_{1}^{2}\left\|A^{1 / 2} v\left(\tau, \theta_{-t-1} \omega\right)\right\|_{2}^{2}\right) d \tau \\
& +c \int_{t}^{t+1}\left(\alpha_{0}^{2}\left\|A^{1 / 2} v\left(\tau, \theta_{-t-1} \omega\right)\right\|_{2}^{2}\right. \\
& \left.\quad+\alpha_{1}^{2}\left\|A v\left(\tau, \theta_{-t-1} \omega\right)\right\|_{2}^{2}\right) d \tau \\
& \quad+c \sum_{j=1}^{n} \int_{t}^{t+1}\left|y_{j}\left(\theta_{\tau-t-1} \omega_{j}\right)\right|^{2} d \tau \\
& \leq c r_{5}(\omega)+c r_{6}(\omega)+c \sum_{j=1}^{n} \int_{-1}^{0}\left|y_{j}\left(\theta_{\tau} \omega_{j}\right)\right|^{2} d \tau \\
& \triangleq r_{7}(\omega) .
\end{aligned}
$$

And then

$$
\begin{aligned}
& \alpha_{0}^{2}\left\|A^{1 / 2} v\left(t+1, \theta_{-t-1} \omega\right)\right\|_{2}^{2}+\alpha_{1}^{2}\left\|A v\left(t+1, \theta_{-t-1} \omega\right)\right\|_{2}^{2} \\
& \leq\left(\alpha_{0}^{2}\left\|A^{1 / 2} v\left(s, \theta_{-t-1} \omega\right)\right\|_{2}^{2}+\alpha_{1}^{2}\left\|A v\left(s, \theta_{-t-1} \omega\right)\right\|_{2}^{2}\right) e^{r_{7}(\omega)} \\
& \quad+c e^{r_{7}(\omega)} r_{5}(\omega)^{2}+c e^{r_{7}(\omega)} \int_{-1}^{0} \sum_{j=1}^{n}\left|y_{j}\left(\theta_{\tau} \omega_{j}\right)\right|^{2} d \tau \\
& +c e^{r_{7}(\omega)} \int_{-1}^{0} \sum_{j=1}^{n}\left|y_{j}\left(\theta_{\tau} \omega_{j}\right)\right|^{4} d \tau \\
& +c e^{r_{7}(\omega)} \int_{t}^{t+1}\|f(\tau)\|_{V^{\prime}}^{2} d \tau \\
& +c e^{r_{7}(\omega)} r_{5}(\omega) \int_{-1}^{0} \sum_{j=1}^{n}\left|y_{j}\left(\theta_{\tau} \omega_{j}\right)\right|^{2} d \tau .
\end{aligned}
$$

Integrating (87) with respect to $s$ over $(t, t+1)$ where $t \geq T_{3}(\omega)$ and employing the property of normal function in (68), we have

$$
\begin{gathered}
\alpha_{0}^{2}\left\|A^{1 / 2} v\left(t+1, \theta_{-t-1} \omega\right)\right\|_{2}^{2}+\alpha_{1}^{2}\left\|A v\left(t+1, \theta_{-t-1} \omega\right)\right\|_{2}^{2} \\
\leq r_{8}(\omega), \quad \forall t \geq T_{3}(\omega), \quad f \in \Sigma\left(f_{0}\right),
\end{gathered}
$$

where

$$
\begin{aligned}
r_{8}(\omega)= & r_{6}(\omega) e^{r_{7}(\omega)}+c e^{r_{7}(\omega)} r_{5}(\omega)^{2} \\
& +c e^{r_{7}(\omega)} \int_{-1}^{0} \sum_{j=1}^{n}\left|y_{j}\left(\theta_{\tau} \omega_{j}\right)\right|^{2} d \tau
\end{aligned}
$$

$$
\begin{aligned}
& +c e^{r_{7}(\omega)} \int_{-1}^{0} \sum_{j=1}^{n}\left|y_{j}\left(\theta_{\tau} \omega_{j}\right)\right|^{4} d \tau \\
& +c e^{r_{7}(\omega)} M_{f_{0}} \\
& +c e^{r_{7}(\omega)} r_{5}(\omega) \int_{-1}^{0} \sum_{j=1}^{n}\left|y_{j}\left(\theta_{\tau} \omega_{j}\right)\right|^{2} d \tau .
\end{aligned}
$$

We define

$$
\begin{aligned}
& \mathscr{K}_{2}(\omega)=\left\{v(\omega) \in D(A) \mid \alpha_{0}^{2}\left\|A^{1 / 2} v(\omega)\right\|_{2}^{2}\right. \\
& \left.+\alpha_{1}^{2}\|A v(\omega)\|_{2}^{2} \leq r_{8}(\omega)\right\} .
\end{aligned}
$$

In conclusion, $\mathscr{K}_{2}(\omega)$ is a uniformly random absorbing set for $\{\mathscr{U}(t, \tau, \omega)\}$ in $D(A)$, which complete the proof.

So much for that we have proved the existence of bounded uniformly (with respect to $f \in \Sigma\left(f_{0}\right)$ ) random absorbing sets in $V$ and $D(A)$. Next we derive the existence of uniformly (with respect to $f \in \Sigma\left(f_{0}\right)$ ) random attractors in $V$ and $D(A)$.

Theorem 17. If $f_{0}(x, s)$ is a normal function in $L_{\text {loc }}^{2}\left(\mathbb{R}, V^{\prime}\right)$, then the process $\left\{U_{f_{0}}(t, \tau, \omega)\right\}$ corresponding to (48) possess a compact uniformly (with respect to $\tau \in \mathbb{R}$ ) random attractor $\mathscr{A}_{1}(\omega)$ in $V$ which coincides with the uniformly (with respect to $\left.f \in \Sigma\left(f_{0}\right)\right)$ random attractor $\mathscr{A}_{\Sigma\left(f_{0}\right)}$ of the family of processes $\left\{\mathscr{U}_{f}(t, \tau, \omega)\right\}, f \in \Sigma\left(f_{0}\right):$

$$
\mathscr{A}_{1}(\omega)=\mathscr{A}_{\Sigma\left(f_{0}\right)}(\omega)=\mathscr{W}_{0, \Sigma\left(f_{0}\right)}\left(\mathscr{K}_{1}\right)=\bigcup_{f \in \Sigma\left(f_{0}\right)} \mathcal{N}_{f}(0, \omega),
$$

where $\mathscr{K}_{1}(\omega)$ is the uniformly (with respect to $f \in \Sigma\left(f_{0}\right)$ ) random absorbing set in $V$ and $\mathcal{N}_{f}(\omega)$ is the kernel of the process $\left\{\mathscr{U}_{f}(t, \tau, \omega)\right\}$. Furthermore, the kernel $\mathcal{N}_{f}(\omega)$ is nonempty for all $f \in \Sigma\left(f_{0}\right)$.

Proof. We only have to verify condition(C). As the previous section, for fixed $m$, let $H_{1}$ be the subspace spanned by $\left\{e_{j}\right\}_{j=1}^{m}$ and $H_{2}$ the orthogonal complement of $H_{1}$ in $H$. We write

$$
v=v_{1}+v_{2}, \quad v_{1} \in H_{1}, \quad v_{2} \in H_{2} \text { for any } v \in H .
$$


The proof of boundary of $v_{1}$ is similar to the proof in Lemma 15. We need to estimate $v_{2}$, where $v=v_{1}+v_{2}$ is a solution of (48). Letting $w=v_{2}$ in (51), we have

$$
\begin{gathered}
\frac{1}{2} \frac{d}{d t}\left(\alpha_{0}^{2}\left\|v_{2}\right\|_{2}^{2}+\alpha_{1}^{2}\left\|A^{1 / 2} v_{2}\right\|_{2}^{2}\right)+v\left(\alpha_{0}^{2}\left\|A^{1 / 2} v_{2}\right\|_{2}^{2}+\alpha_{1}^{2}\left\|A v_{2}\right\|_{2}^{2}\right) \\
=-v\left(\sum_{j=1}^{n} A h_{j} y_{j}\left(\theta_{t} \omega_{j}\right), v_{2}\right) \\
-\left(\widetilde { B } \left(v+z\left(\theta_{t} \omega\right), \alpha_{0}^{2} v+\alpha_{1}^{2} A v\right.\right. \\
\left.\left.\quad+\sum_{j=1}^{n} h_{j} y_{j}\left(\theta_{t} \omega_{j}\right)\right), v_{2}\right) \\
+\left(\operatorname{Pf}(x, t), v_{2}\right)+\mu\left(\sum_{j=1}^{n} h_{j} y_{j}\left(\theta_{t} \omega_{j}\right), v_{2}\right) .
\end{gathered}
$$

Employing Lemma 14, the second term on the right-hand side of (93) can be bounded by

$$
\begin{aligned}
& \|\left(\widetilde { B } \left(v+z\left(\theta_{t} \omega\right), \alpha_{0}^{2} v+\alpha_{1}^{2} A v\right.\right. \\
& \left.\left.\quad+\sum_{j=1}^{n} h_{j} y_{j}\left(\theta_{t} \omega_{j}\right)\right), v_{2}\right) \mid \\
& \leq c\left\|v+z\left(\theta_{t} \omega\right)\right\|_{2}^{1 / 2}\left\|A^{1 / 2} v+A^{1 / 2} z\left(\theta_{t} \omega\right)\right\|_{2}^{1 / 2} \\
& \quad \times\left\|\alpha_{0}^{2} v+\alpha_{1}^{2} A v+\sum_{j=1}^{n} h_{j} y_{j}\left(\theta_{t} \omega_{j}\right)\right\|_{2}\left\|A v_{2}\right\|_{2} \\
& \quad+c\left\|A^{1 / 2} v+A^{1 / 2} z\left(\theta_{t} \omega\right)\right\|_{2} \\
& \quad \times\left\|\alpha_{0}^{2} v+\alpha_{1}^{2} A v+\sum_{j=1}^{n} h_{j} y_{j}\left(\theta_{t} \omega_{j}\right)\right\|_{2} \\
& \quad \times\left\|A^{1 / 2} v_{2}\right\|_{2}^{1 / 2}\left\|A v_{2}\right\|_{2}^{1 / 2} \\
& \leq \frac{v \alpha_{0}^{2}}{8}\left\|A^{1 / 2} v_{2}\right\|_{2}^{2}+\frac{v \alpha_{1}^{2}}{2}\left\|A v_{2}\right\|_{2}^{2} \\
& \quad+c\left(\alpha_{0}^{2}\|v\|_{2}^{2}+\alpha_{1}^{2}\left\|A^{1 / 2} v\right\|_{2}^{2}\right) \\
& \quad \times\left(\alpha_{0}^{2}\left\|A^{1 / 2} v\right\|_{2}^{2}+\alpha_{1}^{2}\|A v\|_{2}^{2}\right) \\
& +c\left(\alpha_{0}^{2}\|v\|_{2}^{2}+\alpha_{1}^{2}\left\|A^{1 / 2} v\right\|_{2}^{2}\right)^{2} \\
& +c\left(\alpha_{0}^{2}\left\|A^{1 / 2} v\right\|_{2}^{2}+\alpha_{1}^{2}\|A v\|_{2}^{2}\right)^{2}
\end{aligned}
$$

$$
\begin{aligned}
& +c \sum_{j=1}^{n}\left|y_{j}\left(\theta_{j} \omega_{j}\right)\right|^{2}+c \sum_{j=1}^{n}\left|y_{j}\left(\theta_{j} \omega_{j}\right)\right|^{4} \\
& +c \sum_{j=1}^{n}\left|y_{j}\left(\theta_{j} \omega_{j}\right)\right|^{2}\left(\alpha_{0}^{2}\|v\|_{2}^{2}+\alpha_{1}^{2}\left\|A^{1 / 2} v\right\|_{2}^{2}\right) \\
& +c \sum_{j=1}^{n}\left|y_{j}\left(\theta_{j} \omega_{j}\right)\right|^{2}\left(\alpha_{0}^{2}\left\|A^{1 / 2} v\right\|_{2}^{2}+\alpha_{1}^{2}\|A v\|_{2}^{2}\right) .
\end{aligned}
$$

By Poincaré's inequality, note that

$$
\begin{gathered}
v\left|\left(\sum_{j=1}^{n} A h_{j} y_{j}\left(\theta_{j} \omega_{j}\right), v_{2}\right)\right| \\
\leq \frac{v \alpha_{0}^{2}}{8}\left\|A^{1 / 2} v_{2}\right\|_{2}^{2}+c \sum_{j=1}^{n}\left|y_{j}\left(\theta_{t} \omega_{j}\right)\right|^{2}, \\
\left|\left(P f, v_{2}\right)\right| \leq\|f\|_{V^{\prime}}\left\|v_{2}\right\| \leq \frac{v \alpha_{0}^{2}}{8}\left\|A^{1 / 2} v_{2}\right\|_{2}^{2}+c\|f\|_{V^{\prime}}^{2}, \\
\mu\left|\left(\sum_{j=1}^{n} h_{j} y_{j}\left(\theta_{t} \omega_{j}\right), v_{2}\right)\right| \\
\leq \frac{\nu \alpha_{0}^{2}}{8}\left\|A^{1 / 2} v_{2}\right\|_{2}^{2}+c \sum_{j=1}^{n}\left|y_{j}\left(\theta_{j} \omega_{j}\right)\right|^{2} .
\end{gathered}
$$

Associating with (93)-(95) and applying Poincaré inequality, we have

$$
\begin{aligned}
& \frac{d}{d t}\left(\alpha_{0}^{2}\left\|v_{2}\right\|_{2}^{2}+\alpha_{1}^{2}\left\|A^{1 / 2} v_{2}\right\|_{2}^{2}\right) \\
& \quad+\nu \lambda_{m+1}\left(\alpha_{0}^{2}\left\|v_{2}\right\|_{2}^{2}+\alpha_{1}^{2}\left\|A^{1 / 2} v_{2}\right\|_{2}^{2}\right) \\
& \leq c\|f\|_{V^{\prime}}^{2}+G(t, \omega),
\end{aligned}
$$

where

$$
\begin{aligned}
& G(t, \omega) \\
& =c\left(\alpha_{0}^{2}\|v\|_{2}^{2}+\alpha_{1}^{2}\left\|A^{1 / 2} v\right\|_{2}^{2}\right)^{2} \\
& +c\left(\alpha_{0}^{2}\left\|A^{1 / 2} v\right\|_{2}^{2}+\alpha_{1}^{2}\|A v\|_{2}^{2}\right)^{2} \\
& +c \sum_{j=1}^{n}\left|y_{j}\left(\theta_{t} \omega_{j}\right)\right|^{2} \\
& +c\left(\alpha_{0}^{2}\|v\|_{2}^{2}+\alpha_{1}^{2}\left\|A^{1 / 2} v\right\|_{2}^{2}\right) \\
& \quad \times\left(\alpha_{0}^{2}\left\|A^{1 / 2} v\right\|_{2}^{2}+\alpha_{1}^{2}\|A v\|_{2}^{2}\right) \\
& +c \sum_{j=1}^{n}\left|y_{j}\left(\theta_{t} \omega_{j}\right)\right|^{4}
\end{aligned}
$$




$$
\begin{aligned}
& +c \sum_{j=1}^{n}\left|y_{j}\left(\theta_{t} \omega_{j}\right)\right|^{2}\left(\alpha_{0}^{2}\|v\|_{2}^{2}+\alpha_{1}^{2}\left\|A^{1 / 2} v\right\|_{2}^{2}\right) \\
& +c \sum_{j=1}^{n}\left|y_{j}\left(\theta_{t} \omega_{j}\right)\right|^{2}\left(\alpha_{0}^{2}\left\|A^{1 / 2} v\right\|_{2}^{2}+\alpha_{1}^{2}\|A v\|_{2}^{2}\right) .
\end{aligned}
$$

Applying Gronwall Lemma on $[t, t+1]$ where $t \geq T_{3}(\omega)$, we have

$$
\begin{aligned}
& \alpha_{0}^{2}\left\|v_{2}(t+1, \omega)\right\|_{2}^{2}+\alpha_{1}^{2}\left\|A^{1 / 2} v_{2}(t+1, \omega)\right\|_{2}^{2} \\
& \leq e^{-\nu \lambda_{m+1}}\left(\alpha_{0}^{2}\left\|v_{2}(t, \omega)\right\|_{2}^{2}+\alpha_{1}^{2}\left\|A^{1 / 2} v_{2}(t, \omega)\right\|_{2}^{2}\right) \\
& \quad+\int_{t}^{t+1} G(s, \omega) e^{-\nu \lambda_{m+1}} d s \\
& \quad+c \int_{t}^{t+1}\|f(s)\|_{V^{\prime}}^{2} e^{-\nu \lambda_{m+1}} d s .
\end{aligned}
$$

Replacing $\omega$ by $\theta_{-t-1} \omega$ in (98), we have

$$
\begin{gathered}
\alpha_{0}^{2}\left\|v_{2}\left(t+1, \theta_{-t-1} \omega\right)\right\|_{2}^{2} \\
+\alpha_{1}^{2}\left\|A^{1 / 2} v_{2}\left(t+1, \theta_{-t-1} \omega\right)\right\|_{2}^{2} \\
\leq e^{-v \lambda_{m+1}}\left(\alpha_{0}^{2}\left\|v_{2}\left(t, \theta_{-t-1} \omega\right)\right\|_{2}^{2}\right. \\
\left.+\alpha_{1}^{2}\left\|A^{1 / 2} v_{2}\left(t, \theta_{-t-1} \omega\right)\right\|_{2}^{2}\right) \\
+\int_{t}^{t+1} G\left(s, \theta_{-t-1} \omega\right) e^{-v \lambda_{m+1}} d s \\
+c \int_{t}^{t+1}\|f(s)\|_{V^{\prime}}^{2} e^{-v \lambda_{m+1}} d s .
\end{gathered}
$$

Now we need to estimate the second term on the right-hand side of (99). As the previous consideration in Lemma 15, for $s \in[t, t+1)$ where $t \geq T_{3}(\omega)$, we have

$$
\alpha_{0}^{2}\left\|v\left(s, \theta_{-t-2}\right)\right\|_{2}^{2}+\alpha_{1}^{2}\left\|A^{1 / 2} v\left(s, \theta_{-t-2}\right)\right\|_{2}^{2} \leq r_{9}(\omega),
$$

where $r_{9}(\omega)=e^{2 \nu \lambda_{1}} r_{4}(\omega)$. As the previous consideration in Lemma 16, we have

$$
\begin{aligned}
\int_{t}^{t+1}( & \alpha_{0}^{2}\left\|A^{1 / 2} v\left(s, \theta_{-t-2} \omega\right)\right\|_{2}^{2} \\
& \left.+\alpha_{1}^{2}\left\|A v\left(s, \theta_{-t-2} \omega\right)\right\|_{2}^{2}\right) d s \leq r_{10}(\omega),
\end{aligned}
$$

where

$$
\begin{aligned}
r_{10}(\omega)= & c r_{9}(\omega) \sum_{j=1}^{n} \int_{-2}^{0}\left|y_{j}\left(\theta_{s} \omega_{j}\right)\right| d s \\
& +c r_{9}(\omega) \sum_{j=1}^{n} \int_{-2}^{0}\left|y_{j}\left(\theta_{s} \omega_{j}\right)\right|^{2} d s \\
& +c \sum_{j=1}^{n} \int_{-2}^{0}\left|y_{j}\left(\theta_{s} \omega_{j}\right)\right|^{2} d s+r_{9}(\omega) .
\end{aligned}
$$

Replacing $\omega$ by $\theta_{-t-2} \omega$ in (84), we have

$$
\begin{aligned}
& \alpha_{0}^{2}\left\|A^{1 / 2} v\left(t+1, \theta_{-t-2} \omega\right)\right\|_{2}^{2}+\alpha_{1}^{2}\left\|A v\left(t+1, \theta_{-t-2} \omega\right)\right\|_{2}^{2} \\
& \leq\left(\alpha_{0}^{2}\left\|A^{1 / 2} v\left(s, \theta_{-t-2} \omega\right)\right\|_{2}^{2}\right. \\
& \left.+\alpha_{1}^{2}\left\|A v\left(s, \theta_{-t-2} \omega\right)\right\|_{2}^{2}\right) e^{J_{2}\left(s, t+1, \theta_{-t-2} \omega\right)} \\
& +c \int_{t}^{t+1} \sum_{j=1}^{n}\left|y_{j}\left(\theta_{\tau-t-2} \omega_{j}\right)\right|^{2} e^{J_{2}\left(\tau, t+1, \theta_{-t-2} \omega\right)} d \tau \\
& +c \int_{t}^{t+1} \sum_{j=1}^{n}\left|y_{j}\left(\theta_{\tau-t-2} \omega_{j}\right)\right|^{4} e^{J_{2}\left(\tau, t+1, \theta_{-t-2} \omega\right)} d \tau \\
& +c \int_{t}^{t+1} \sum_{j=1}^{n}\left|y_{j}\left(\theta_{\tau-t-2} \omega_{j}\right)\right|^{2} \\
& \quad \times\left(\alpha_{0}^{2}\left\|v\left(\tau, \theta_{-t-2} \omega\right)\right\|_{2}^{2}\right. \\
& \left.+\alpha_{1}^{2}\left\|A^{1 / 2} v\left(\tau, \theta_{-t-2} \omega\right)\right\|_{2}^{2}\right) e^{J_{2}\left(\tau, t+1, \theta_{-t-2} \omega\right)} d \tau \\
& +c \int_{t}^{t+1}\left(\alpha_{0}^{2}\left\|v\left(\tau, \theta_{-t-2} \omega\right)\right\|_{2}^{2}\right. \\
& \left.+\alpha_{1}^{2}\left\|A^{1 / 2} v\left(\tau, \theta_{-t-2} \omega\right)\right\|_{2}^{2}\right)^{2} e^{J_{2}\left(\tau, t+1, \theta_{-t-2} \omega\right)} d \tau \\
& +c \int_{t}^{t+1}\|f(\tau)\|_{V^{\prime}}^{2} e^{J_{2}\left(\tau, t+1, \theta_{-t-2} \omega\right)} d \tau .
\end{aligned}
$$

Analogously to the previous estimates, we have

$$
\begin{gathered}
J_{2}\left(\tau, t+1, \theta_{-t-2} \omega\right) \\
\leq c \int_{t}^{t+1}\left(\alpha_{0}^{2}\left\|v\left(\tau, \theta_{-t-2} \omega\right)\right\|_{2}^{2}\right. \\
\left.+\alpha_{1}^{2}\left\|A^{1 / 2} v\left(\tau, \theta_{-t-2} \omega\right)\right\|_{2}^{2}\right) d \tau \\
+c \int_{t}^{t+1}\left(\alpha_{0}^{2}\left\|A^{1 / 2} v\left(\tau, \theta_{-t-2} \omega\right)\right\|_{2}^{2}\right. \\
\left.+\alpha_{1}^{2}\left\|A v\left(\tau, \theta_{-t-2} \omega\right)\right\|_{2}^{2}\right) d \tau \\
+c \sum_{j=1}^{n} \int_{t}^{t+1}\left|y_{j}\left(\theta_{\tau-t-2} \omega_{j}\right)\right|^{2} d \tau \\
\leq r_{11}(\omega),
\end{gathered}
$$

where

$$
r_{11}(\omega)=c r_{9}(\omega)+c r_{10}(\omega)+c \sum_{j=1}^{n} \int_{-2}^{0}\left|y_{j}\left(\theta_{\tau} \omega_{j}\right)\right|^{2} d \tau
$$


Integrating (103) with respect to $s \in(t, t+1)$ associating (100), (101), and (104) with (68), we have

$$
\begin{aligned}
& \alpha_{0}^{2}\left\|A^{1 / 2} v\left(t+1, \theta_{-t-2} \omega\right)\right\|_{2}^{2}+\alpha_{1}^{2}\left\|A v\left(t+1, \theta_{-t-2} \omega\right)\right\|_{2}^{2} \\
& \leq r_{12}(\omega),
\end{aligned}
$$

where

$$
\begin{aligned}
r_{12}(\omega)= & c r_{10}(\omega) e^{r_{11}(\omega)} \\
& +c e^{r_{11}(\omega)} \int_{-1}^{0} \sum_{j=1}^{n}\left|y_{j}\left(\theta_{\tau} \omega_{j}\right)\right|^{2} d \tau \\
& +c e^{r_{11}(\omega)} \int_{-1}^{0} \sum_{j=1}^{n}\left|y_{j}\left(\theta_{\tau} \omega_{j}\right)\right|^{4} d \tau \\
& +c r_{9}(\omega) e^{r_{11}(\omega)} \int_{-1}^{0} \sum_{j=1}^{n}\left|y_{j}\left(\theta_{\tau} \omega_{j}\right)\right|^{2} d \tau \\
& +c r_{9}(\omega)^{2} e^{r_{11}(\omega)}+c M_{f_{0}} e^{r_{11}(\omega)}
\end{aligned}
$$

Replacing $s, t+1$, and $\omega$ by $t, s$, and $\theta_{-t-1} \omega$, respectively, in (84) where $s \in(t, t+1)$ and $t \geq T_{3}(\omega)$, we have

$$
\begin{aligned}
& \alpha_{0}^{2}\left\|A^{1 / 2} v\left(s, \theta_{-t-1} \omega\right)\right\|_{2}^{2}+\alpha_{1}^{2}\left\|A v\left(s, \theta_{-t-1} \omega\right)\right\|_{2}^{2} \\
& \leq\left(\alpha_{0}^{2}\left\|A^{1 / 2} v\left(t, \theta_{-t-1} \omega\right)\right\|_{2}^{2}\right. \\
& \left.+\alpha_{1}^{2}\left\|A v\left(t, \theta_{-t-1} \omega\right)\right\|_{2}^{2}\right) e^{J_{2}\left(t, t+1, \theta_{-t-1} \omega\right)} \\
& +c \int_{t}^{t+1} \sum_{j=1}^{n}\left|y_{j}\left(\theta_{\tau-t-1} \omega_{j}\right)\right|^{2} e^{J_{2}\left(\tau, t+1, \theta_{-t-1} \omega\right)} d \tau \\
& +c \int_{t}^{t+1} \sum_{j=1}^{n}\left|y_{j}\left(\theta_{\tau-t-1} \omega_{j}\right)\right|^{4} e^{J_{2}\left(\tau, t+1, \theta_{-t-1} \omega\right)} d \tau \\
& +c \int_{t}^{t+1} \sum_{j=1}^{n}\left|y_{j}\left(\theta_{\tau-t-1} \omega_{j}\right)\right|^{2} \\
& \quad \times\left(\alpha_{0}^{2}\left\|v\left(\tau, \theta_{-t-1} \omega\right)\right\|_{2}^{2}\right. \\
& +c \int_{t}^{t+1}\|f(\tau)\|_{V^{\prime}}^{2} e^{J_{2}\left(\tau, t+1, \theta_{-t-1} \omega\right)} d \tau \\
& +c \int_{t}^{t+1}\left(\alpha_{1}^{2}\left\|A^{1 / 2} v\left(\tau, \theta_{-t-1} \omega\right)\right\|_{2}^{2}\right) e^{J_{2}\left(\tau, t+1, \theta_{-t-1} \omega\right)} d \tau \\
& +\alpha_{1}^{2}\left\|A^{1 / 2} v\left(\tau, \theta_{-t-1} \omega\right)\right\|_{2}^{2}
\end{aligned}
$$

$$
\begin{aligned}
& \leq r_{12}(\omega) e^{r_{7}(\omega)} \\
& \quad+c e^{r_{7}(\omega)} \int_{-1}^{0} \sum_{j=1}^{n}\left|y_{j}\left(\theta_{\tau} \omega_{j}\right)\right|^{2} d \tau \\
& \quad+c e^{r_{7}(\omega)} \int_{-1}^{0} \sum_{j=1}^{n}\left|y_{j}\left(\theta_{\tau} \omega_{j}\right)\right|^{4} d \tau+c e^{r_{7}(\omega)} M_{f_{0}} \\
& +c r_{5}(\omega)^{2} e^{r_{7}(\omega)} \\
& +c r_{5}(\omega) e^{r_{7}(\omega)} \int_{-1}^{0} \sum_{j=1}^{n}\left|y_{j}\left(\theta_{\tau} \omega_{j}\right)\right|^{2} d \tau \\
& \triangleq r_{13}(\omega) .
\end{aligned}
$$

Associating with the above inequalities, we have

$$
\int_{t}^{t+1} G\left(s, \theta_{-t-1} \omega\right) d s \leq r_{14}(\omega),
$$

where

$$
\begin{aligned}
r_{14}(\omega)= & c r_{5}(\omega)^{2}+c r_{13}(\omega)^{2} \\
& +c \int_{-1}^{0} \sum_{j=1}^{n}\left|y_{j}\left(\theta_{s} \omega_{j}\right)\right|^{2} d s \\
& +c r_{5}(\omega) r_{13}(\omega) \\
& +c \int_{-1}^{0} \sum_{j=1}^{n}\left|y_{j}\left(\theta_{s} \omega_{j}\right)\right|^{4} d s \\
& +c r_{5}(\omega) \int_{-1}^{0} \sum_{j=1}^{n}\left|y_{j}\left(\theta_{s} \omega_{j}\right)\right|^{2} d s \\
& +c r_{13}(\omega) \int_{-1}^{0} \sum_{j=1}^{n}\left|y_{j}\left(\theta_{s} \omega_{j}\right)\right|^{2} d s .
\end{aligned}
$$

According to the property of $\left\{\lambda_{j}\right\}_{j=1}^{\infty}$ mentioned in Section 3, for $\mathbb{P}$ a.e $\omega \in \Omega$ and $\forall \varepsilon>0$, there exists $M$ such that for $m \geq M$

$$
\begin{gathered}
e^{-v \lambda_{m+1}}\left(\alpha_{0}^{2}\left\|v_{2}\left(t, \theta_{-t-1} \omega\right)\right\|_{2}^{2}+\alpha_{1}^{2}\left\|A^{1 / 2} v_{2}\left(t, \theta_{-t-1} \omega\right)\right\|_{2}^{2}\right) \\
\leq \frac{r_{5}(\omega)}{e^{\nu \lambda_{m+1}}} \leq \varepsilon r_{5}(\omega), \\
\int_{t}^{t+1} G\left(s, \theta_{-t-1} \omega\right) e^{-v \lambda_{m+1}} d s \leq \frac{r_{14}(\omega)}{e^{\nu \lambda_{m+1}}} \leq \varepsilon r_{14}(\omega), \\
c \int_{t}^{t+1}\|f(s)\|_{V^{\prime}}^{2} e^{-v \lambda_{m+1}} d s \leq \frac{c M_{f_{0}}}{e^{\nu \lambda_{m+1}}} \leq \varepsilon .
\end{gathered}
$$


Therefore, we deduce from (99) that, for $\mathbb{P}$ a.e. $\omega \in \Omega$,

$$
\begin{aligned}
& \alpha_{0}^{2}\left\|v_{2}\left(t, \theta_{-t} \omega\right)\right\|_{2}^{2}+\alpha_{1}^{2}\left\|A^{1 / 2} v_{2}\left(t, \theta_{-t} \omega\right)\right\|_{2}^{2} \\
& \quad \leq \varepsilon+\varepsilon r_{5}(\omega)+\varepsilon r_{14}(\omega), \quad \forall t \geq T_{3}(\omega), \quad f \in \Sigma\left(f_{0}\right),
\end{aligned}
$$

which indicates $\left\{\mathscr{U}_{f}(t, \tau, \omega)\right\}, f \in \Sigma\left(f_{0}\right)$ satisfying uniform (with respect to $f \in \Sigma\left(f_{0}\right)$ ) condition(C) in $V$. According to Theorem 12, the proof is completed.

In the following we prove the existence of uniformly random attractor for the families of processes $\left\{\mathcal{U}_{f}(t, \tau, \omega)\right\}$, $f \in \Sigma\left(f_{0}\right)$ corresponding to $(48)$ in $D(A)$.

Theorem 18. If $f_{0}(x, s)$ is a normal function in $L_{\mathrm{loc}}^{2}\left(\mathbb{R}, V^{\prime}\right)$, then the process $\left\{\mathcal{U}_{f_{0}}(t, \tau, \omega)\right\}$ corresponding to (48) possess a compact uniformly (with respect to $\tau \in \mathbb{R}$ ) random attractor $\mathscr{A}_{2}(\omega)$ in $D(A)$ which coincides with the uniformly (with respect to $f \in \Sigma\left(f_{0}\right)$ ) random attractor $\mathscr{A}_{\Sigma\left(f_{0}\right)}$ of the family of processes $\left\{\mathcal{U}_{f}(t, \tau, \omega)\right\}, f \in \Sigma\left(f_{0}\right)$ :

$$
\mathscr{A}_{2}(\omega)=\mathscr{A}_{\Sigma\left(f_{0}\right)}(\omega)=\mathscr{W}_{0, \Sigma\left(f_{0}\right)}\left(\mathscr{K}_{2}\right)=\bigcup_{f \in \Sigma\left(f_{0}\right)} \mathcal{N}_{f}(0, \omega)
$$

where $\mathscr{K}_{2}(\omega)$ is the uniformly (with respect to $f \in \Sigma\left(f_{0}\right)$ ) random absorbing set in $D(A)$ and $\mathcal{N}_{f}(\omega)$ is the kernel of the process $\left\{\mathcal{U}_{f}(t, \tau, \omega)\right\}$. Furthermore, the kernel $\mathcal{N}_{f}(\omega)$ is nonempty for all $f \in \Sigma\left(f_{0}\right)$.

Proof. In Lemma 16, we have proved that the semigroup of processes $\left\{\mathcal{U}_{f}(t, \tau, \omega)\right\}, f \in \Sigma\left(f_{0}\right)$, has a uniformly random absorbing set in $D(A)$. Now we testify that the semigroup of processes corresponding to (48) satisfies uniform (with respect to $f \in \Sigma\left(f_{0}\right)$ ) condition (C). Analogously to the proof in Lemma 16, we easily check that $v_{1}$ is bounded in $D(A)$. Letting $w=A v_{2}$ in (51), we have

$$
\begin{gathered}
\frac{1}{2} \frac{d}{d t}\left(\alpha_{0}^{2}\left\|A^{1 / 2} v_{2}\right\|_{2}^{2}+\alpha_{1}^{2}\left\|A v_{2}\right\|_{2}^{2}\right) \\
+v\left(\alpha_{0}^{2}\left\|A v_{2}\right\|_{2}^{2}+\alpha_{1}^{2}\left\|A^{3 / 2} v_{2}\right\|_{2}^{2}\right) \\
=-v\left(\sum_{j=1}^{n} A h_{j} y_{j}\left(\theta_{t} \omega_{j}\right), A v_{2}\right) \\
-\left(\begin{array}{c}
v+z\left(\theta_{t} \omega\right), \alpha_{0}^{2} v+\alpha_{1}^{2} A v \\
\left.+\sum_{j=1}^{n} h_{j} y_{j}\left(\theta_{t} \omega_{j}\right)\right), A v_{2}
\end{array}\right)
\end{gathered}
$$

$$
\begin{aligned}
& +\left(\operatorname{Pf}(x, t), A v_{2}\right) \\
& +\mu\left(\sum_{j=1}^{n} h_{j} y_{j}\left(\theta_{t} \omega_{j}\right), A v_{2}\right) .
\end{aligned}
$$

Applying Lemma 14, the second term on the right-hand side of (114) can be bounded by

$$
\begin{aligned}
& \mid\left(\widetilde { B } \left(v+z\left(\theta_{t} \omega\right), \alpha_{0}^{2} v+\alpha_{1}^{2} A v\right.\right. \\
& \left.\left.\quad+\sum_{j=1}^{n} h_{j} y_{j}\left(\theta_{t} \omega_{j}\right)\right), A v_{2}\right) \mid \\
& \leq \frac{v \alpha_{0}^{2}}{8}\left\|A v_{2}\right\|_{2}^{2}+\frac{v \alpha_{1}^{2}}{2}\left\|A^{3 / 2} v_{2}\right\|_{2}^{2} \\
& \quad+c\left(\alpha_{0}^{2}\|v\|_{2}^{2}+\alpha_{1}^{2}\left\|A^{1 / 2} v\right\|_{2}^{2}\right) \\
& \quad \times\left(\alpha_{0}^{2}\left\|A^{1 / 2} v\right\|_{2}^{2}+\alpha_{1}^{2}\|A v\|_{2}^{2}\right) \\
& \quad+c\left(\alpha_{0}^{2}\|v\|_{2}^{2}+\alpha_{1}^{2}\left\|A^{1 / 2} v\right\|_{2}^{2}\right)^{2} \\
& +c \sum_{j=1}^{n}\left|y_{j}\left(\theta_{t} \omega_{j}\right)\right|^{2}\left(\alpha_{0}^{2}\|v\|_{2}^{2}+\alpha_{1}^{2}\left\|A^{1 / 2} v\right\|_{2}^{2}\right)^{2} \\
& +c\left(\alpha_{0}^{2}\left\|A^{1 / 2} v\right\|_{2}^{2}+\alpha_{1}^{2}\|A v\|_{2}^{2}\right)^{2} \\
& +c \sum_{j=1}^{n}\left|y_{j}\left(\theta_{t} \omega_{j}\right)\right|^{2}+c \sum_{j=1}^{n}\left|y_{j}\left(\theta_{t}^{2} \omega_{j}\right)\right|^{4}
\end{aligned}
$$

Note that

$$
\begin{gathered}
v\left|\left(\sum_{j=1}^{n} h_{j} y_{j}\left(\theta_{t} \omega_{j}\right), A v_{2}\right)\right| \leq \frac{v \alpha_{0}^{2}}{8}\left\|A v_{2}\right\|_{2}^{2}+c \sum_{j=1}^{n}\left|y_{j}\left(\theta_{t} \omega_{j}\right)\right|^{2}, \\
\left|\left(P f(x, t), A v_{2}\right)\right| \leq\|f\|_{V^{\prime}}\left\|A v_{2}\right\|_{2} \leq \frac{v \alpha_{0}^{2}}{8}\left\|A v_{2}\right\|_{2}^{2}+c\|f\|_{V^{\prime}}^{2}, \\
\mu\left|\left(\sum_{j=1}^{n} h_{j} y_{j}\left(\theta_{t} \omega_{j}\right), A v_{2}\right)\right| \leq \frac{v \alpha_{0}^{2}}{8}\left\|A v_{2}\right\|_{2}^{2}+c \sum_{j=1}^{n}\left|y_{j}\left(\theta_{t} \omega_{j}\right)\right|^{2} .
\end{gathered}
$$


Associating with (114)-(116) and applying Poincaré's inequality, we have

$$
\begin{aligned}
& \frac{d}{d t}\left(\alpha_{0}^{2}\left\|A^{1 / 2} v_{2}\right\|_{2}^{2}+\alpha_{1}^{2}\left\|A v_{2}\right\|_{2}^{2}\right) \\
& \quad+v \lambda_{m+1}\left(\alpha_{0}^{2}\left\|A^{1 / 2} v_{2}\right\|_{2}^{2}+\alpha_{1}^{2}\left\|A v_{2}\right\|_{2}^{2}\right) \\
& \leq G(t, \omega)+c\|f\|_{V^{\prime}}^{2} .
\end{aligned}
$$

Applying Gronwall's lemma over $s \in[t, t+1]$ where $t \geq T_{2}(\omega)$, we have

$$
\begin{aligned}
\alpha_{0}^{2}\left\|A^{1 / 2} v_{2}(t+1, \omega)\right\|_{2}^{2}+\alpha_{1}^{2}\left\|A v_{2}(t+1, \omega)\right\|_{2}^{2} \\
\leq\left(\alpha_{0}^{2}\left\|A^{1 / 2} v_{2}(t, \omega)\right\|_{2}^{2}+\alpha_{1}^{2}\left\|A v_{2}(t, \omega)\right\|_{2}^{2}\right) e^{-\gamma \lambda_{m+1}} \\
\quad+\int_{t}^{t+1} G(s, \omega) e^{-\nu \lambda_{m+1}} d s+c \int_{t}^{t+1}\|f\|_{V^{\prime}}^{2} e^{-\nu \lambda_{m+1}} d s .
\end{aligned}
$$

Replacing $\omega$ by $\theta_{-t-1} \omega$ in (118), we have

$$
\begin{gathered}
\alpha_{0}^{2}\left\|A^{1 / 2} v_{2}\left(t+1, \theta_{-\mathrm{t}-1} \omega\right)\right\|_{2}^{2} \\
+\alpha_{1}^{2}\left\|A v_{2}\left(t+1, \theta_{-t-1} \omega\right)\right\|_{2}^{2} \\
\leq\left(\alpha_{0}^{2}\left\|A^{1 / 2} v_{2}\left(t, \theta_{-t-1} \omega\right)\right\|_{2}^{2}\right. \\
\left.+\alpha_{1}^{2}\left\|A v_{2}\left(t, \theta_{-t-1} \omega\right)\right\|_{2}^{2}\right) e^{-v \lambda_{m+1}} \\
+\int_{t}^{t+1} G\left(s, \theta_{-t-1} \omega\right) e^{-\nu \lambda_{m+1}} d s \\
+c \int_{t}^{t+1}\|f\|_{V^{\prime}}^{2} e^{-v \lambda_{m+1}} d s .
\end{gathered}
$$

Analogously to the consideration in Theorem 17 , for $\mathbb{P}$ a.e. $\omega \in \Omega$ and $\forall \varepsilon>0$, there exists $M$ such that, for $m>M$,

$$
\begin{gathered}
e^{-v \lambda_{m+1}}\left(\alpha_{0}^{2}\left\|A^{1 / 2} v_{2}\left(t, \theta_{-t-1} \omega\right)\right\|_{2}^{2}+\alpha_{1}^{2}\left\|A v_{2}\left(t, \theta_{-t-1} \omega\right)\right\|_{2}^{2}\right) \\
\leq \frac{r_{12}(\omega)}{e^{\nu \lambda_{m+1}}} \leq \varepsilon r_{12}(\omega), \\
\int_{t}^{t+1} G\left(s, \theta_{-t-1} \omega\right) e^{-v \lambda_{m+1}} d s \leq \frac{r_{14}(\omega)}{e^{\nu \lambda_{m+1}}} \leq \varepsilon r_{14}(\omega), \\
\quad c \int_{t}^{t+1}\|f(s)\|_{V^{\prime}}^{2} e^{-\nu \lambda_{m+1}} d s \leq \frac{c M_{f_{0}}}{e^{\nu \lambda_{m+1}}} \leq \varepsilon .
\end{gathered}
$$

Therefore, we deduce from (119) that, for $\mathbb{P}$ a.e $\omega \in \Omega$,

$$
\begin{gathered}
\alpha_{0}^{2}\left\|A^{1 / 2} v_{2}\left(t+1, \theta_{-t-1} \omega\right)\right\|_{2}^{2}+\alpha_{1}^{2}\left\|A v_{2}\left(t+1, \theta_{-t-1} \omega\right)\right\|_{2}^{2} \\
\leq \varepsilon+\varepsilon r_{12}(\omega)+\varepsilon r_{14}(\omega), \quad \forall t \geq T_{3}(\omega), \quad f \in \Sigma\left(f_{0}\right) .
\end{gathered}
$$

which indicates $\left\{\mathscr{U}_{f}(t, \tau, \omega)\right\}, f \in \Sigma\left(f_{0}\right)$ satisfying uniform (with respect to $f \in \Sigma\left(f_{0}\right)$ ) condition $(\mathrm{C})$ in $D(A)$. According to Theorem 12, the proof is completed.

Now we introduce a homeomorphism $\Phi\left(\theta_{t} \omega\right) u=u+$ $z\left(\theta_{t} \omega\right), u \in E$, whose inverse homeomorphism $\Phi^{-1}\left(\theta_{t} \omega\right) u=$ $u-z\left(\theta_{t} \omega\right)$. Then the transformation

$$
\mathscr{V}_{\sigma}(t, \tau, \omega)=\Phi\left(\theta_{t} \omega\right) \circ \mathscr{U}_{\sigma}(t, \tau, \omega) \circ \Phi^{-1}\left(\theta_{\tau} \omega\right)
$$

generates a process corresponding to (8). Note that the two processes are equivalent by (122). It is easy to check that $\mathscr{V}_{\sigma}(t, \tau, \omega)$ has a uniformly (with respect to $\sigma \in \Sigma$ ) random attractor provided $\mathcal{U}_{\sigma}(t, \tau, \omega)$ possesses a uniformly (with respect to $\sigma \in \Sigma$ ) random attractor. As a result Theorem 17 and Theorem 18 imply that (8) has a uniformly (with respect to $\left.f \in \Sigma\left(f_{0}\right)\right)$ random attractor in $V$ and $D(A)$.

\section{Acknowledgments}

The authors would like to thank the reviewers for the valuable suggestions and comments. The work is supported by the National Natural Science Foundation (no. 11171115).

\section{References}

[1] R. Camassa and D. D. Holm, "An integrable shallow water equation with peaked solitons," Physical Review Letters, vol. 71, no. 11, pp. 1661-1664, 1993.

[2] R. Camassa, D. D. Holm, and J. M. Hyman, "An new integrable shallow water equation," Advances in Applied Mechanics, vol. 31, pp. 1-33, 1994.

[3] H. R. Dullin, G. A. Gottwald, and D. D. Holm, "On asymptotically equivalent shallow water wave equations," Physica $D$, vol. 190, no. 1-2, pp. 1-14, 2004.

[4] H. R. Dullin, G. A. Gottwald, and D. D. Holm, "Camassa-Holm, Korteweg-de Vries-5 and other asymptotically equivalent equations for shallow water waves," Fluid Dynamics Research, vol. 33, no. 1-2, pp. 73-95, 2003.

[5] A. Constantin and W. A. Strauss, "Stability of the CamassaHolm solitons," Journal of Nonlinear Science, vol. 12, no. 4, pp. 415-422, 2002.

[6] A. Bressan and A. Constantin, "Global conservative solutions of the Camassa-Holm equation," Archive for Rational Mechanics and Analysis, vol. 183, no. 2, pp. 215-239, 2007.

[7] A. Constantin and W. A. Strauss, "Stability of peakons," Communications on Pure and Applied Mathematics, vol. 53, no. 5, pp. 603-610, 2000.

[8] A. Constantin and J. Escher, "Global existence and blow-up for a shallow water equation," Annali della Scuola Normale Superiore di Pisa, vol. 26, no. 2, pp. 303-328, 1998.

[9] G. Rodríguez-Blanco, "On the Cauchy problem for the Camassa-Holm equation," Nonlinear Analysis, vol. 46, no. 3, pp. 309-327, 2001.

[10] A. Constantin and J. Escher, "Wave breaking for nonlinear nonlocal shallow water equations," Acta Mathematica, vol. 181, no. 2, pp. 229-243, 1998.

[11] Z. Xin and P. Zhang, "On the weak solutions to a shallow water equation," Communications on Pure and Applied Mathematics, vol. 53, no. 11, pp. 1411-1433, 2000. 
[12] A. Bressan and A. Constantin, "Global dissipative solutions of the Camassa-Holm equation," Analysis and Applications, vol. 5, no. 1, pp. 1-27, 2007.

[13] Y. Long, Z. Li, and W. Rui, "New travelling wave solutions for a nonlinearly dispersive wave equation of Camassa-Holm equation type," Applied Mathematics and Computation, vol. 217, no. 4, pp. 1315-1320, 2010.

[14] E. A. Olson, "Well posedness for a higher order modified Camassa-Holm equation," Journal of Differential Equations, vol. 246, no. 10, pp. 4154-4172, 2009.

[15] L. Tian, C. Shen, and D. Ding, "Optimal control of the viscous Camassa-Holm equation," Nonlinear Analysis, vol. 10, no. 1, pp. 519-530, 2009.

[16] D. Henry, "Infinite propagation speed for a two component Camassa-Holm equation," Discrete and Continuous Dynamical Systems B, vol. 12, no. 3, pp. 597-606, 2009.

[17] J. Escher, O. Lechtenfeld, and Z. Yin, "Well-posedness and blowup phenomena for the 2-component Camassa-Holm equation," Discrete and Continuous Dynamical Systems A, vol. 19, no. 3, pp. 493-513, 2007.

[18] J. B. Li and Y. S. Li, "Bifurcations of travelling wave solutions for a two-component Camassa-Holm equation," Acta Mathematica Sinica, vol. 24, no. 8, pp. 1319-1330, 2008.

[19] C. Foias, D. D. Holm, and E. S. Titi, "The three dimensional viscous Camassa-Holm equations, and their relation to the Navier-Stokes equations and turbulence theory," Journal of Dynamics and Differential Equations, vol. 14, no. 1, pp. 1-35, 2002.

[20] T. Caraballo, A. M. Márquez-Durán, and J. Real, “The asymptotic behaviour of a stochastic 3D LANS- $\alpha$ model," Applied Mathematics and Optimization, vol. 53, no. 2, pp. 141-161, 2006.

[21] T. Caraballo, A. M. Márquez-Durán, and J. Real, "Pullback and forward attractors for a 3D LANS- $\alpha$ model with delay," Discrete and Continuous Dynamical Systems A, vol. 15, no. 2, pp. 559-578, 2006.

[22] D. Wu, "Finite dimensional uniform attractors for the nonautonomous Camassa-Holm equations," Abstract and Applied Analysis, vol. 2009, Article ID 952657, 15 pages, 2009.

[23] T. Tachim Medjo, "Averaging of a 3D Lagrangian averaged Navier-Stokes- $\alpha$ model with oscillating external forces," Communications on Pure and Applied Analysis, vol. 10, no. 4, pp. 1281-1305, 2011.

[24] T. Tachim Medjo, "A non-autonomous 3D Lagrangian averaged Navier-Stokes- $\alpha$ model with oscillating external force and its global attractor," Communications on Pure and Applied Analysis, vol. 10, no. 2, pp. 415-433, 2011.

[25] A. V. Babin and M. I. Vishik, Attractors of Evolution Equations, North-Holland, Amsterdam, The Netherlands, 1992.

[26] A. Haraux, Systèmes Dynamiques Dissipatifs et Applications, Masson, Paris, France, 1991.

[27] V. V. Chepyzhov and M. I. Vishik, Attractors for Equations of Mathematical Physics, American Mathematical Society, Providence, RI, USA, 2002.

[28] L. Arnold, Random Dynamical Systems, Springer, Berlin, Germany, 1998.

[29] H. Crauel and F. Flandoli, "Attractors for random dynamical systems," Probability Theory and Related Fields, vol. 100, no. 3, pp. 365-393, 1994.

[30] I. Chueshov, Monotone Random Systems Theory and Applications, Springer, Berlin, Germany, 2002.
[31] G. Da Prato and J. Zabczyk, Stochastic Equations in Infinite Dimensions, Cambridge University Press, Cambridge, UK, 1992.

[32] B. Øksendal, Stochastic Differential Equations, Springer, Berlin, Germany, 2000.

[33] H. Crauel, A. Debussche, and F. Flandoli, "Random attractors," Journal of Dynamics and Differential Equations, vol. 9, no. 2, pp. 307-341, 1997.

[34] F. Flandoli and B. Schmalfuss, "Random attractors for the 3D stochastic Navier-Stokes equation with multiplicative white noise," Stochastics and Stochastics Reports, vol. 59, no. 1-2, pp. 21-45, 1996.

[35] J. K. Hale, Asymptotic Behavior of Dissipative Systems, American Mathematical Society, Providence, RI, USA, 1988.

[36] J. C. Robinson, Infinite-Dimensional Dynamical Systems, Cambridge University Press, Cambridge, UK, 2001.

[37] G. R. Sell and Y. You, Dynamics of Evolutionary Equations, Springer, New York, NY, USA, 2002.

[38] R. Teman, Infinite-Dimensional Dynamical Systems in Machanics and Physics, Springer, New York, NY, USA, 1998.

[39] A. O. Çelebi, V. K. Kalantarov, and M. Polat, "Attractors for the generalized Benjamin-Bona-Mahony equation," Journal of Differential Equations, vol. 157, no. 2, pp. 439-451, 1999.

[40] F. Morillas and J. Valero, "Attractors for reaction-diffusion equations in $\mathbb{R}^{n}$ with continuous nonlinearity," Asymptotic Analysis, vol. 44, no. 1-2, pp. 111-130, 2005.

[41] S. Lu, H. Wu, and C. Zhong, "Attractors for nonautonomous 2D Navier-Stokes equations with normal external forces," Discrete and Continuous Dynamical Systems A, vol. 13, no. 3, pp. 701-719, 2005.

[42] O. Ladyzhenskaya, Attractors for Semigroups and Evolution Equations, Cambridge University Press, Cambridge, UK, 1991.

[43] P. W. Bates, K. Lu, and B. Wang, "Attractors for lattice dynamical systems," International Journal of Bifurcation and Chaos in Applied Sciences and Engineering, vol. 11, no. 1, pp. 143-153, 2001.

[44] P. W. Bates, H. Lisei, and K. Lu, "Attractors for stochastic lattice dynamical systems," Stochastics and Dynamics, vol. 6, no. 1, pp. 1-21, 2006.

[45] P. W. Bates, K. Lu, and B. Wang, "Random attractors for stochastic reaction-diffusion equations on unbounded domains," Journal of Differential Equations, vol. 246, no. 2, pp. 845-869, 2009.

[46] B. Wang, "Random attractors for the stochastic BenjaminBona-Mahony equation on unbounded domains," Journal of Differential Equations, vol. 246, no. 6, pp. 2506-2537, 2009.

[47] X. Han, W. Shen, and S. Zhou, "Random attractors for stochastic lattice dynamical systems in weighted spaces," Journal of Differential Equations, vol. 250, no. 3, pp. 1235-1266, 2011.

[48] Z. Wang, S. Zhou, and A. Gu, "Random attractor of the stochastic strongly damped wave equation," Communications in Nonlinear Science and Numerical Simulation, vol. 17, no. 4, pp. 1649-1658, 2012.

[49] Z. Wang, S. Zhou, and A. Gu, "Random attractor for a stochastic damped wave equation with multiplicative noise on unbounded domains," Nonlinear Analysis, vol. 12, no. 6, pp. 3468-3482, 2011. 


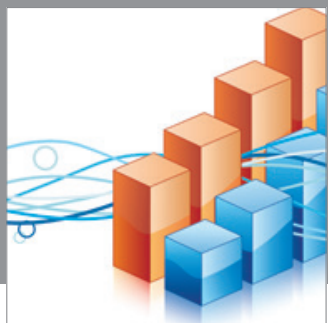

Advances in

Operations Research

mansans

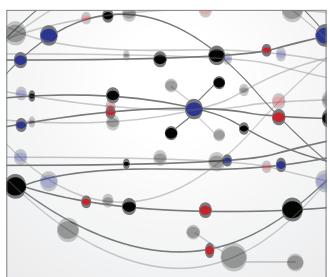

The Scientific World Journal
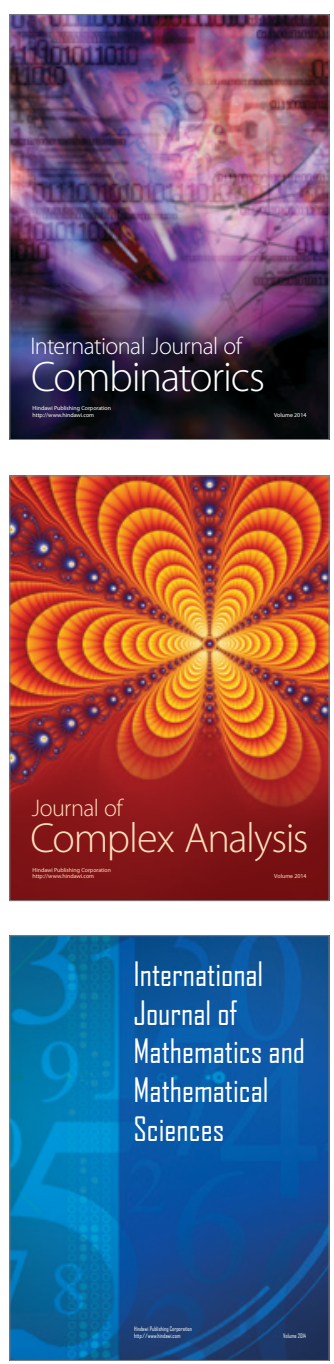
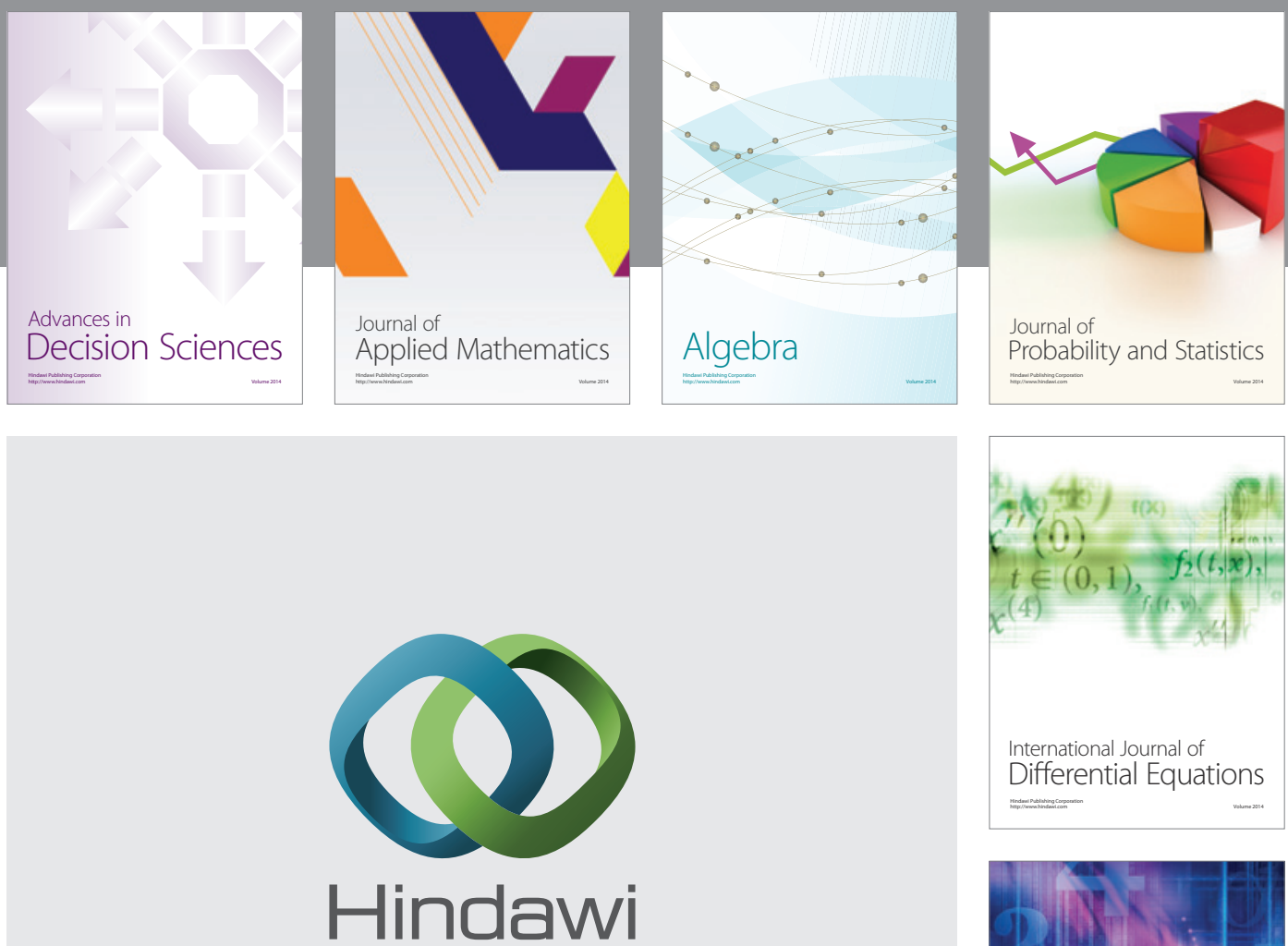

Submit your manuscripts at http://www.hindawi.com
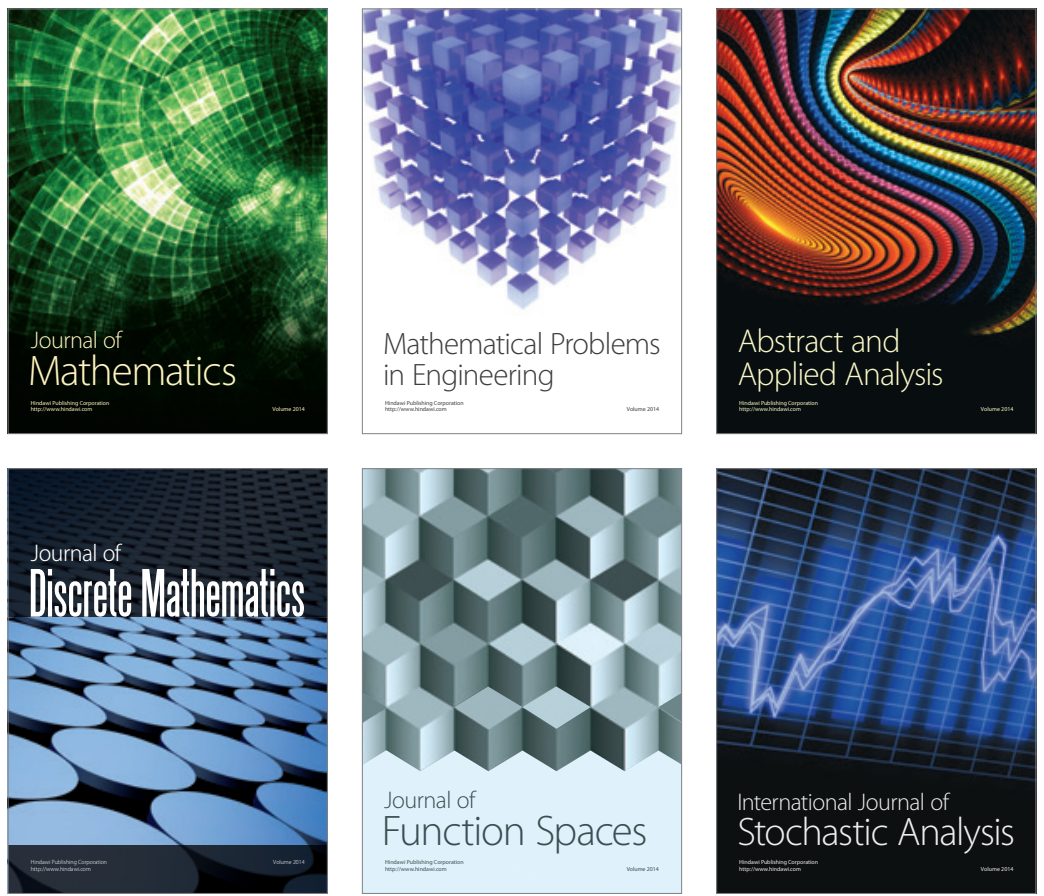

Journal of

Function Spaces

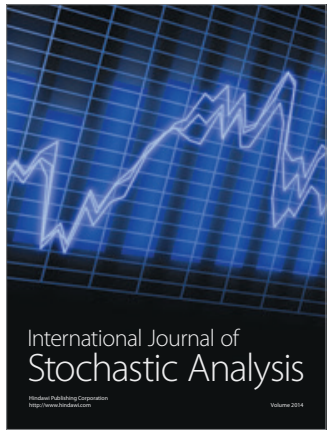

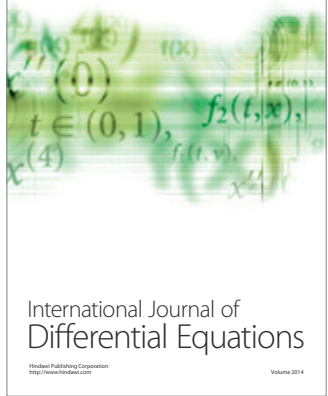
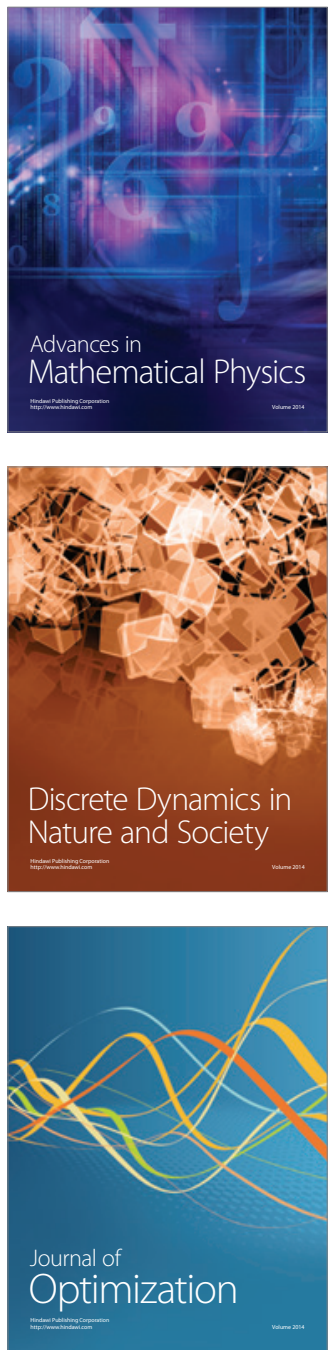